\title{
Effect of different parameters on mixture formation and flow field in simulations of an evaporative spray injection test case
}

\author{
Mateus Dias Ribeiro ${ }^{1}$ Alex Mendonça Bimbato ${ }^{1} \cdot$ Maurício Araújo Zanardi ${ }^{1}$ José Antônio Perrella Balestieri ${ }^{1}$
}

Received: 25 April 2017 / Accepted: 10 September 2017 / Published online: 23 April 2018

(c) The Brazilian Society of Mechanical Sciences and Engineering 2018

\begin{abstract}
Direct injection plays an important role in the efforts to increase efficiency of modern engines, and the correct evaluation of the velocity and fuel mixture fraction fields is crucial for modeling combustion in fuel sprays. Therefore, a computational study has been performed to assess the effect of different parameters on the mixture formation and flow field in the simulation of a single jet of the engine combustion network (ECN) "Spray G" evaporative gasoline injection test case. The Lagrangian particle tracking (LPT) approach was tested within both Reynolds-averaged Navier-Stokes (RANS) and large eddy simulation (LES) frameworks, and the varieties were compared. Additional parameters that were considered include mesh resolution $(0.75,0.50$, and $0.25 \mathrm{~mm}$ ) and droplet breakup (Reitz-Diwakar, Reitz-KHRT, and Pilch-Erdman), as well as stochastic turbulent dispersion (O'Rourke) and stochastic collision (O'Rourke) models. Experimental penetration length data from both liquid and vapor phases were used to validate the 54 simulations performed within this study. Then, a series of analyses were performed to weigh the effect of each isolated parameter on the outcome of the simulations. Finally, three additional simulations were conducted to study specific issues of LES in fuel spray modeling. In this way, this study was able to make a qualitative comparison of the evaporative spray cloud shapes and the evaluation of spray statistics in terms of the iso-octane mixture fraction and droplet/slip velocities.
\end{abstract}

Keywords Fuel spray $\cdot$ ICE $\cdot$ RANS $\cdot$ LES $\cdot$ Lagrangian particle tracking

\section{Introduction}

Worldwide legislation on greenhouse gases and pollutant emissions has become much stricter in recent years. As an example, projections for the European $\mathrm{CO}_{2}$ emissions have a target fleet average of $95 \mathrm{~g} / \mathrm{km}$ for all new passenger cars by 2021, while the United States Environmental Protection Agency (EPA) has committed to an average of $93 \mathrm{~g} / \mathrm{km}$ by 2025 [1]. To achieve these ambitious goals, new technologies must be developed to improve the efficiency and emissions of the new generation of passenger cars. Direct injection spark ignition (DISI) engines are one of these

Technical Editor: Jader Barbosa Jr.

Mateus Dias Ribeiro

mateusdiasbr@gmail.com

1 School of Engineering, São Paulo State University (UNESP), Guaratinguetá Campus, Av. Dr. Ariberto Pereira da Cunha, 333 Portal das Colinas, Guaratinguetá, SP CEP 12.516-410, Brazil technologies. They are of crucial importance to the reduction of specific fuel consumption and to the achievement of these strict emission standards in the stateof-the-art internal combustion engines [2, 3].

This improved efficiency is made possible by the direct injection of the fuel into the combustion chamber during the intake or in compression stroke at very high injection pressures. If the injection is made within the compression stroke, the spray cloud formed at the injector nozzle has a very narrow window of time during which it can exchange heat with the gas in the combustion chamber, evaporate, and mix with the carrier phase. This process induces the formation of a region of very high fuel concentration in the vicinity of the spark plug and close to the longitudinal axis of the spray. The longer the distance from the spray core, the lower the fuel concentration will be. This concentration ranges from rich to stoichiometric and lean limits. Combustion in such an environment is known as stratified charge combustion because ignitable fuel concentrations are expected to be present only in a small region of the combustion chamber until ignition is triggered. In this case, 
lower specific fuel consumption can be obtained in comparison to stoichiometric fuel conditions, as in the case of conventional spark ignition engines, since now a large volume within the combustion chamber has a very low fuel concentration or no fuel at all. Furthermore, direct injection also eliminates load throttling and minimizes pumping losses; the evaporation of fuel droplets cools down the charge inside the chamber, enabling an increase in the compression ratio [4].

In case of higher loads, however, elevated pressure and strong turbulent mean fluctuations may cause the spray cloud to collapse, and a compact ignitable region next to the spark plug will be harder to achieve [5]. In this case, a stoichiometric or fuel-rich mixture may be necessary, and it can be achieved with an early injection during the intake stroke. Thus, the injected fuel mass has enough time to mix adequately inside the engine. For instance, Yang and Anderson [6] obtained improved full-load torque output in a DISI engine using a combined approach with late injection under part load and early injection under full load to combine the volumetric efficiency of the early injection with the suppression of the late injection's tendency to knock. In another study, Zheng et al. [7] reported that a $25 \%$ ratio (in the case of late injections) and a $75 \%$ ratio (in the case of early injections) may be a reasonable strategy for low-speed operations (up to $1200 \mathrm{rpm}$ ), with the development of a local, ignitable, and rich mixture close to the spark plug leading to higher thermal efficiency and lower soot emissions.

The understanding of the physical phenomena involved in fuel sprays, such as atomization, droplet breakup, collision, heat transfer, momentum transfer and evaporation, is of high importance for the development of direct injection engines. To understand them, techniques have been developed to measure important spray parameters in experimental test benches such as stationary combustion chambers and optical engines [3, 8, 9]. Most of these techniques, however, rely on optical devices such as laser apparatuses that are still limited in their ability to capture important regions such as the dense spray close to the nozzle. Furthermore, due to the windows with limited optical accessibility in such experimental test benches, big portions of the domain may not be reachable. To circumvent those problems, computational methods involving fuel sprays can be employed. These methods have evolved rapidly in recent years, largely due to the increase in computational power.

A comprehensive review of the history of computational methods applied to combustion since the 1950s has been presented by Westbrook et al. [10]. In it, they note the progress in the modeling of fluid dynamics, chemical kinetics, sprays, and turbulence. A myriad of works have been published using different methods and computational codes to simulate evaporative/non-evaporative fuel sprays and spray combustion techniques [11-15]. Most of these studies have employed the Lagrangian particle tracking (LPT) approach to describe the liquid phase and have combined it with either a Reynolds-averaged NavierStokes (RANS) model or a large eddy simulation (LES) framework to simulate turbulence in the gas phase. Further reviews of the LPT strategy can be found in [16] and [17].

Many academic contributions have been made to the development of more efficient and cleaner spark ignited engines with conventional indirect injection systems [18-23] but only a few publications at this time have addressed the study of DISI engines, which may potentially improve fuel consumption and emissions when compared to their conventionally injected counterparts. Therefore, in this study, we wish to simulate the ECN Spray G test case that corresponds to a non-reacting early injection case for spray-guided gasoline engines. Moreover, we have performed a computational study on the effect of different parameters (mesh resolution, turbulence, collision, turbulent dispersion, and secondary breakup) on mixture formation and flow field. Details on the Spray G condition and information on the structure of the 54 simulations performed within this work are described in Sect. 4. In the next two sections, we describe the spray modeling and all of its relevant phenomena in two main sections: modeling of the gas phase and modeling of the particle phase.

\section{Modeling of the gas phase}

This study uses Lagrangian-Eulerian methodology to model injection, breakup, heat transfer, vaporization, and the mixture process of a gasoline spray with the carrier phase. The environment (pressure and temperature) is set to be similar to an engine combustion chamber at the beginning of the compression stroke. Therefore, the gas phase is assumed to be continuous, and an Eulerian approach is used for its representation. For this, two different frameworks were tested to model turbulence: the first uses a RANS approach, and the second uses an LES approach.

In the RANS approach, the instantaneous quantities of the flow are decomposed into their time-averaged and fluctuating parts, and the whole set of governing equations of the flow (continuity, momentum, and energy) are averaged. Only the mean quantities of the flow are resolved, since the average of each fluctuating property is assumed equal to zero and is therefore neglected. However, the Reynolds stress term (RST) does not vanish and must be modeled. When dealing with compressible flows, a change in variable is often applied for every quantity of interest $\varphi$, in which $\varphi$ is density weighted by the operation $\bar{\rho} \tilde{\varphi}=\overline{\rho \varphi}$. This is known as Favre filtering and results in the Favrefiltered variable $\tilde{\varphi}$. In the case of RANS, $\tilde{\varphi}$ represents a 
Favre filter of the averaged part of a quantity (such as the velocity $u$ ). The whole set of the RANS equations for mass (1), momentum (2), energy (3), and species (4) conservation in the Favre-filtered form reads

$$
\begin{aligned}
& \frac{\partial \bar{\rho}}{\partial t}+\frac{\partial\left(\bar{\rho} \tilde{u_{i}}\right)}{\partial x_{i}}=S_{\rho} \\
& \frac{\partial\left(\bar{\rho} \tilde{u_{i}}\right)}{\partial t}+\frac{\partial\left(\bar{\rho} \tilde{u_{i}} \tilde{u_{j}}\right)}{\partial x_{j}} \\
& =-\frac{\partial \bar{p}}{\partial x_{i}}+\frac{\partial}{\partial x_{j}}\left[\bar{\rho} \bar{v}\left(\frac{\partial \tilde{u_{j}}}{\partial x_{i}}+\frac{\partial \tilde{u_{i}}}{\partial x_{j}}\right)-\frac{2}{3} \bar{\rho} \bar{v} \frac{\partial \tilde{u_{k}}}{\partial x_{k}} \delta_{i j}-\frac{\partial}{\partial x_{j}}\left(\overline{\rho u_{i}{ }^{\prime \prime} u_{k}{ }^{\prime \prime}}\right)\right] \\
& \quad+\bar{\rho} g_{i}+S_{u},
\end{aligned}
$$

$$
\begin{gathered}
\frac{\partial(\bar{\rho} \tilde{h})}{\partial t}+\frac{\partial\left(\bar{\rho} \tilde{h} \tilde{u_{j}}\right)}{\partial x_{j}}+\frac{\partial(\bar{\rho} \tilde{K})}{\partial t}+\frac{\partial\left(\bar{\rho} \tilde{K} \tilde{u_{j}}\right)}{\partial x_{j}} \\
=\frac{\partial}{\partial x_{j}}\left(\alpha_{e f f} \frac{\partial \tilde{h}}{\partial x_{j}}\right)+\frac{\partial \bar{p}}{\partial t}+S_{h}, \\
\frac{\partial\left(\bar{\rho} \tilde{Y}_{i}\right)}{\partial t}+\frac{\partial\left(\bar{\rho} \tilde{Y}_{i} \tilde{u}_{j}\right)}{\partial x_{j}}=\frac{\partial}{\partial x_{j}}\left(\mu_{e f f} \frac{\partial \tilde{Y}}{\partial x_{j}}\right)+S_{Y_{i}} .
\end{gathered}
$$

The RST term $-\rho \mathrm{u}_{\mathrm{i}}{ }^{\prime \prime} \mathrm{u}_{\mathrm{k}}{ }^{\prime \prime}$ is modeled with the well-established $k-\epsilon$ model. In the previous equations, $u$ represents the velocity (in three spatial coordinates $i, j$ and $k$ ), $\rho$ is the fluid density, $v$ is the kinematic viscosity, $\mu_{\text {eff }}$ is the effective viscosity, $\alpha_{\text {eff }}$ is the effective thermal diffusivity, $p$ is the pressure, $h$ is the enthalpy, $K$ is the kinetic energy, $g$ is the acceleration of gravity, $Y_{i}$ is the mixture fraction of species $\mathrm{i}$ and $S_{\rho}, S_{\mathrm{u}}, S_{\mathrm{h}}$ and $S_{\mathrm{Y}_{\mathrm{i}}}$ are the source terms needed to couple the gas phase with the particle phase. Finally, $u_{i}{ }^{\prime \prime}$ represents the fluctuating part of the velocity $u_{i}$, and the bar symbol over the variables represents the averages.

In an LES, however, both mean and fluctuating parts are resolved up to a certain limit, which defines a filter for what must be carried out (by the Navier-Stokes equations) and what must be modeled. This filter separates the large scales from the small scales of the given flow. Most of the turbulent kinetic energy is resolved, whereas the unresolved subgrid scales are modeled by one subgrid model. In this case, the momentum equation is described by Eq. 5, but now $\tilde{\varphi}$ represents the Favre filter of the absolute value for the quantity of interest, i.e., resolved filtered mean and fluctuating parts, with the bar symbol now representing LES filtering rather than averaging. The same aspects apply to the other governing equations.

$$
\begin{aligned}
& \frac{\partial\left(\bar{\rho} \tilde{u_{i}}\right)}{\partial t}+\frac{\partial\left(\bar{\rho} \tilde{u_{i}} \tilde{u_{j}}\right)}{\partial x_{j}} \\
& =\frac{\partial}{\partial x_{j}}\left[\bar{\rho} \bar{v}\left(\frac{\partial \tilde{u_{j}}}{\partial x_{i}}+\frac{\partial \tilde{u_{i}}}{\partial x_{j}}\right)-\frac{2}{3} \bar{\rho} \bar{\nu} \frac{\partial \tilde{u_{k}}}{\partial x_{k}} \delta_{i j}-\bar{\rho} \tau_{i j}{ }^{s g s}\right] \\
& \quad-\frac{\partial \bar{p}}{\partial x_{i}}+\bar{\rho} g_{i}+S_{u} .
\end{aligned}
$$

In the above equation, $\tau_{\mathrm{ij}}{ }^{\text {sgs }}$ represents the subgrid turbulence scales, modeled here using the standard Smagorinsky model $\left(C_{\mathrm{S}}=0.168[24]\right)$.

\section{Modeling of the particle phase}

For modeling the liquid phase, which is composed of many discrete tiny droplets or particles, Lagrangian methodology was employed, and the sub-models used to represent each phenomenon of the simulated full-cone spray (Fig. 1) are described further. The parcel approach was used in this study to reduce the computational effort. Thus, to circumvent the difficult problem of simulating each particle separately, one can define a parcel as group of droplets with the same properties. In this way, only bins of particles need to be treated numerically. Another reasonable assumption is to consider that all particles are perfectly spherical, with a diameter $d$. The size distribution of the injected droplets is modeled by the Rosin-Rammler cumulative density function (CDF), as shown in Eq. (6),

$$
F(x, n, d)=1-e^{-(x / d)^{n}}
$$

where $n$ is the constant of uniformity, $d$ is the characteristic particle size, and $x$ is a particular particle size within the distribution.

The velocity magnitude of the injected parcels can be determined either by knowing the pressure drop between the injector nozzle and combustion chamber and applying

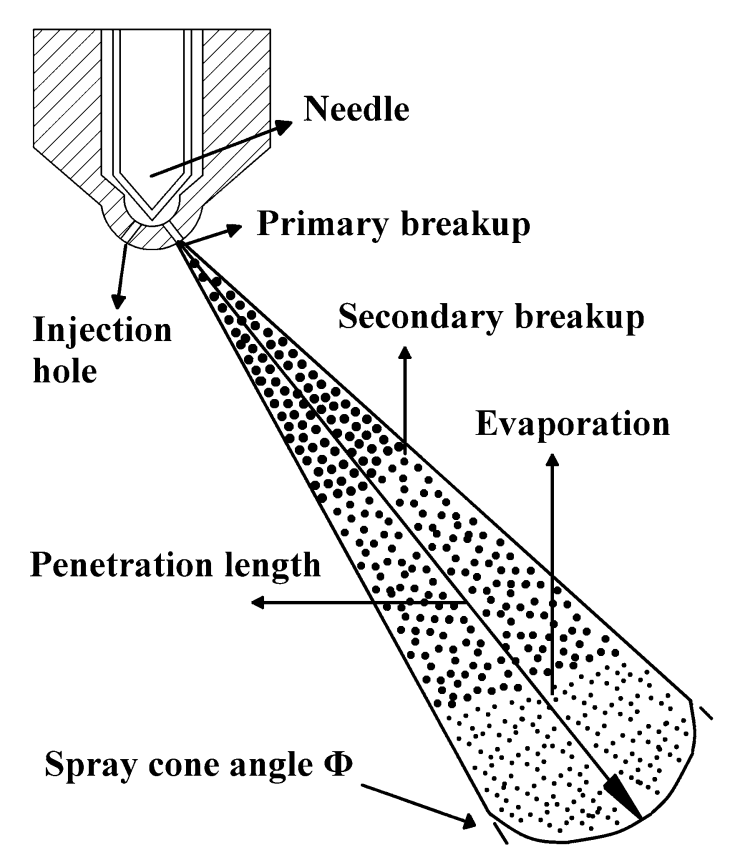

Fig. 1 Schematic representation of a full-cone spray and the different phenomena undergone by a fuel spray during injection 
the Bernoulli equation or by deriving it from the mass flow rate and injector geometry properties. Their directions are correlated with expressions that distribute the parcels over a predefined range, in which small particles tend to be injected within an angle (not greater than a maximum predefined value) and the larger particles tend to go straight or to stay within a small angle. When liquid fuel particles are injected at high velocities in a quiescent gas environment, they are decelerated by the gaseous environment. This exchange of momentum between the liquid and gas phases takes place due to the relative velocity between the two. Equation (7), as shown in [11], denotes the exchange of momentum by evaluating the resulting drag force over a liquid parcel moving with relative velocity $u_{\text {res }}$ with respect to the gas phase,

$\frac{1}{6} \rho_{P} \pi d^{3} \frac{\mathrm{d} u_{P}}{\mathrm{~d} t}=\frac{1}{2}\left(u_{\mathrm{g}}-u_{\mathrm{P}}\right)\left|u_{\mathrm{g}}-u_{\mathrm{P}}\right| \rho_{\mathrm{g}} C_{\mathrm{d}} \frac{\pi d^{2}}{4}$.

The term on the left side in Eq. (7) represents the change in the acceleration of the liquid fuel particle, while the term on the right side denotes the drag force due to its relative motion to the gas phase. The subscript $g$ refers to the gas phase and the subscript $P$ refers to the liquid parcel of diameter $d$.

The drag coefficient $C_{\mathrm{D}}$ is calculated assuming spherical particles using the Reynolds number of the gas phase $\mathrm{Re}_{\mathrm{g}}=\mathrm{du}_{\mathrm{rel}} \rho_{\mathrm{g}} / \mu_{\mathrm{g}}$. The correlation for evaluating $C_{\mathrm{D}}$ is presented in Eq. (8),

$$
\begin{aligned}
C_{\mathrm{D}}=\frac{24}{R e_{g}}\left(1+\frac{1}{6} \mathrm{Re}_{\mathrm{g}}{ }^{2 / 3}\right), & \mathrm{Re} & \leq 1000 \\
0.424, & \operatorname{Re} & >1000 .
\end{aligned}
$$

Finally, the parcels can be tracked by updating their positions according to Eq. (9),

$\frac{\mathrm{d} x_{\mathrm{P}}}{\mathrm{d} t}=u_{\mathrm{P}}$.

As soon as the liquid particles get into the domain, they exchange not only momentum, but also heat with the gas phase. The liquid phase exchanges heat with the gas phase due to diffusive and convective transport and radiation. The heat exchange increases the temperature until the particle reaches the boiling point and evaporates. This is very important for the mixing process, combustion, and formation of pollutants, since the fuel does not start to burn until it is in the vapor phase.

In addition to the assumption of perfect spherical particles, one may also neglect the effect of radiation, since it is small compared to that of convection. Furthermore, the evaporation modeling is based on the average flow field around the particles, because the calculation of the flow among them is not feasible. The energy balance for a liquid particle [4] is presented in Eq. (10),

$$
m_{\mathrm{P}} c_{\mathrm{p}, 1} \frac{\mathrm{d} T_{\mathrm{P}}}{\mathrm{d} t}+\Delta h_{\text {evap }} \frac{\mathrm{d} m_{\text {evap }}}{\mathrm{d} t}=\lambda_{\mathrm{g}} \pi d_{\mathrm{P}}\left(T_{\infty}-T_{\mathrm{P}}\right) \frac{\zeta}{e^{\zeta}-1} \mathrm{Nu}
$$

The terms $\mathrm{Nu}$ and $\zeta$ are shown in Eq. (11) and Eq. (12), respectively,

$$
\begin{aligned}
& \mathrm{Nu}=\frac{h d_{\mathrm{P}}}{\lambda_{\mathrm{g}}}, \\
& \zeta=\frac{\dot{m}_{\text {evap }} c_{\mathrm{p}, \text { vap }}}{\mathrm{Nu} \lambda_{\mathrm{g}} \pi d_{\mathrm{P}}} .
\end{aligned}
$$

In the previous equations, $T_{\infty}$ is the temperature of the gas phase and $T_{\mathrm{P}}$ is the temperature of the parcel, while $d_{\mathrm{P}}$ is the parcel diameter, $h$ is the heat transfer coefficient at the parcel surface, $\lambda_{\mathrm{g}}$ is the thermal conductivity of the gas mixture, $\mathrm{Nu}$ is the Nusselt number and $\zeta$ is a dimensionless correction factor accounting for the reduced heat transfer due to the simultaneous mass transfer from the parcel to the gas phase. In Eq. (10), the first term represents the energy necessary to heat the liquid fuel parcel to a given temperature in an interval $\mathrm{d} t$, where $m_{\mathrm{p}}$ is the mass of the parcel, $T_{\mathrm{P}}$ is its temperature, and $c_{\mathrm{p}, 1}$ is the specific heat at a constant liquid fuel pressure. The second term accounts for the energy necessary to evaporate the parcel mass $m_{\text {evap }}$ during the same time interval. The term on the right-hand side accounts for the heat transfer between the liquid and gas phase.

The appropriate Nusselt number that accounts for the relative velocity between the particle and gas is evaluated using the heat transfer correlation proposed by Ranz and Marshall [25], and Eq. (13) reads

$$
\mathrm{Nu}=2.0+0.6 \operatorname{Re}^{1 / 2} \operatorname{Pr}^{1 / 3} \text {. }
$$

In this equation, the Reynolds number is denoted as Re and the Prandtl number is shown as Pr, both of which are calculated using the properties of the gas phase.

Finally, droplet vaporization is calculated as described by Zuo et al. [26] and taking fuel droplet superheat vaporization into account. The model distinguishes between two kinds of vaporization: vaporization due to flash boiling (calculated with Adachi's [27] experimental correlation) and vaporization due to normal heat transfer with the gaseous phase. The rate of vaporizing mass is giving by $\mathrm{G}$ in Eq. (14),

$$
G=4 \pi \frac{k}{c_{\mathrm{p}}} r_{0} \frac{1}{1+G_{\mathrm{f}} / G} \ln \left[1+\left(1+\frac{G_{\mathrm{f}}}{G}\right) \frac{h_{\infty}-h_{\mathrm{b}}}{L\left(T_{\mathrm{b}}\right)}\right]
$$

where $G_{\mathrm{f}}$ is the flash boiled vapor rate, $k$ and $c_{\mathrm{p}}$ are the gaseous thermal conductivity and specific heat, respectively, $r_{0}$ is the droplet radius, $h_{\infty}$ and $h_{\mathrm{b}}$ are the gaseous enthalpies in the mixture and at the drop surface, 
respectively, and $L\left(T_{\mathrm{b}}\right)$ is the latent heat at the local boiling temperature.

\subsection{Droplet breakup}

The formation of droplets from a round continuous liquid jet can be distinguished into four different breakup regimes, as shown by Reitz and Bracco [28]: the Rayleigh regime, the first and second wind-induced regimes, and the atomization regime. The formation of these first droplets is known as primary breakup, and in the case of fuel sprays, atomization is the governing regime. These droplets are then subject of secondary breakup, i.e., new particles are formed from the collapse of previous ones, due to the high relative velocities in the liquid-gas interface. Baumgarten [4] presents five different liquid drop breakup regimes: vibrational breakup (1), bag breakup (2), bag/ streamer breakup (3), stripping breakup (4), and catastrophic breakup (5). These regimes are defined by Wierzba [29] (see Fig. 2).

The relative velocity in the liquid-gas interface induces aerodynamic forces that create oscillations on the droplet surface. The increase in amplitude of these oscillations results in droplet breakup. The Weber number $\mathrm{We}_{\mathrm{P}}=$ $u^{2}{ }_{\text {rel }} D \rho_{\mathrm{g}} / \sigma$ is the relevant parameter for the liquid drop breakup mechanism. This number is a relation between aerodynamic force (which tends to break up droplets into smaller particles) and surface tension (which tries to keep the parent droplets in their spherical shape). The symbol $u_{\text {rel }}$ is the relative velocity between particles and gas, $D$ is

Fig. 2 Droplet breakup regimes according to Wierzba [29] the particle diameter, $\rho_{\mathrm{g}}$ is the gas density, and $\sigma$ is the droplet surface tension. As the droplets become smaller, the surface tension necessary to break them up becomes bigger, and therefore, the relative velocity must also be bigger to disintegrate the drop.

Experimental investigations have shown that distinct breakup regimes can be divided according to a range of Weber numbers. Table 1 presents the transition Weber numbers for different breakup regimes. Several models have been proposed in the literature for the numerical simulation of secondary breakup process. In the present study, three secondary breakup models were tested: the Reitz-Diwakar model [30, 31], the Reitz-KHRT (KelvinHelmholtz/Rayleigh-Taylor) model [32], and the PilchErdman model [33].

\subsubsection{Reitz-Diwakar secondary breakup model}

The Reitz-Diwakar breakup model [31] works based on two different assumptions. The first is that atomization and drop breakup near the nozzle within the spray cloud are considered to be indistinguishable; thus the blob method is used to represent the jet breakup into the first spray droplets. In this model, a detailed simulation of the phenomena near the nozzle is replaced by the injection of large spherical droplets, which are uniform in diameter and similar in size to the nozzle hole. The large droplets that are injected are then subject to secondary breakup as they travel through the gaseous medium.

\section{2 - Bag breakup}

1 - Vibrational breakup

3 - Bag/streamer breakup

4 - Stripping breakup

5 - Catastrophic breakup
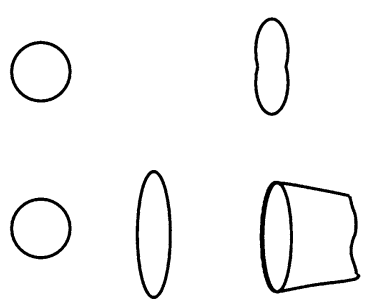

$\sqrt{1} \because:$

$\therefore \vdots$
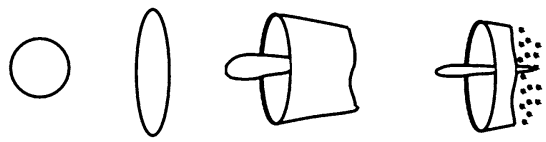

$\therefore \because$
$\because \because \because$
$\because \because$
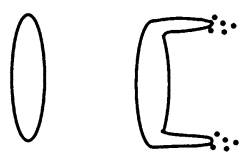<smiles>CC=CCC</smiles>
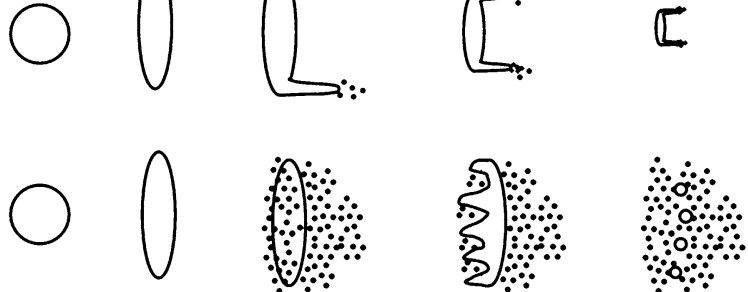
Table 1 Transition Weber numbers for different regimes, as in Baumgarten [4]

\begin{tabular}{llll}
\hline Wierzba [29] & Weber number & Arcoumanis et al. [34] & Weber number \\
\hline 1. Vibrational & $\approx 12$ & 1. Vibrational & $\approx 12$ \\
2. Bag & $<20$ & 2. Bag & $<18$ \\
3. Bag/streamer & $<50$ & 3. Bag/streamer & $<45$ \\
4. Stripping & $<100$ & 4. Chaotic breakup & $<100$ \\
5. Catastrophic & $>100$ & 5. Sheet stripping & $<350$ \\
& & 6. Wave crest stripping & $<1000$ \\
& & 7. Catastrophic & $>1000$ \\
\hline
\end{tabular}

The second assumption is that secondary breakup can be distinguished between two droplet regimes: the bag breakup and the stripping breakup. Bag breakup will occur only if $\mathrm{We}_{\mathrm{P}}$ is greater than a certain value, which is defined to be the bag constant ( 0.75 for RANS and 0.15 for LES). For higher $u_{\text {rel }}$, stripping breakup may occur when We $>C_{\text {strip }} \sqrt{\mathrm{Re}}$, where $C_{\text {strip }}=0.5$ is the stripping constant. Regardless of the breakup regime, the reduction in the particle radius is described by Eq. 15, where $r_{\mathrm{P}}$ is the particle radius and $r_{\text {stable }}$ is the new stable particle radius after breakup,

$\frac{\mathrm{d} r_{\mathrm{P}}}{\mathrm{d} t}=\frac{-\left(r_{\mathrm{P}}-r_{\text {stable }}\right)}{\tau_{\mathrm{br}}}$.

The characteristic breakup times $\tau_{\text {br }}$ for bag and stripping breakup are given by Eq. 16 , where $C_{1}=0.785$ and $C_{2}=$ 10 are the constant models. The variables $\rho_{\mathrm{P}}$ and $\rho_{\mathrm{g}}$ represent liquid density and gas density, respectively,

$$
\begin{aligned}
& \tau_{\text {br-bag }}=C_{1} \sqrt{\frac{\rho_{\mathrm{P}} r_{\mathrm{P}}^{3}}{2 \sigma}} \\
& \tau_{\text {br-stripping }}=C_{2} \frac{r}{u_{\text {rel }}} \sqrt{\frac{\rho_{\mathrm{P}}}{\rho_{\mathrm{g}}} .}
\end{aligned}
$$

The new stable particle radius after breakup is given by Eq. 17 for both bag breakup and stripping breakup,

$$
\begin{aligned}
& r_{\text {stable-bag }}=\frac{6 \sigma}{\rho_{\mathrm{g}} u_{\mathrm{rel}}^{2}} C_{1} \\
& r_{\text {stable-stripping }}=\frac{\sigma^{2}}{2 \rho_{\mathrm{g}}^{2} u_{\mathrm{rel}^{3}} v} .
\end{aligned}
$$

\subsubsection{Reitz-KHRT secondary breakup model}

Beale and Reitz [32] postulate that droplet breakup is caused by the increase in waves on its surface. The model can be applied to both diesel and gasoline sprays in two distinct steps. In the first step, primary breakup is predicted by the Kelvin-Helmholtz $(\mathrm{KH})$ instability model, and the diameters of the first droplets ("blobs") are derived. In the second step, secondary breakup is predicted with the Kelvin-Helmholtz instability model in conjunction with the Rayleigh-Taylor (RT) accelerative instability model.
The $\mathrm{KH}$ frequency and $\mathrm{KH}$ wavelength of the fastestgrowing wave are shown in Eq. 18 , where $Z=\sqrt{\mathrm{We}_{1}} / \mathrm{Re}_{1}$ is the Ohnesorge number, $T=Z \sqrt{\mathrm{We}_{\mathrm{g}}}$ is the Taylor number, $\mathrm{Re}_{1}$ is the liquid Reynolds number, $\mathrm{We}_{1}$ is the liquid Weber number, and $\mathrm{We}_{\mathrm{g}}$ is the gas Weber number,

$$
\begin{aligned}
& \Omega_{\mathrm{KH}} \frac{0.34+0.38 \mathrm{We}_{\mathrm{g}}^{1.5}}{(1+Z)\left(1+1.4 T^{0.6}\right)} \sqrt{\frac{\sigma}{\rho_{\mathrm{P}} r^{3}}} \\
& \Lambda_{\mathrm{KH}}=\frac{9.02 r(1+0.45 \sqrt{Z})\left(1+0.4 T^{0.7}\right)}{\left(1+0.865 \mathrm{We}_{\mathrm{g}}{ }^{1.67}\right)^{0.6}} .
\end{aligned}
$$

The RT instability is also a wave instability on the drop surface, and the frequency of its fastest-growing wave is given by Eq. 19, where $g_{\mathrm{T}}$ is the acceleration in the direction of travel. The corresponding wave number $K_{\mathrm{RT}}$ is also shown in Eq. 19,

$$
\begin{aligned}
& \Omega_{\mathrm{RT}}=\sqrt{\frac{2}{3 \sqrt{3 \sigma}} \frac{\left[-g_{\mathrm{T}}\left(\rho_{\mathrm{P}}-\rho_{\mathrm{g}}\right)\right]^{3 / 2}}{\rho_{\mathrm{P}}+\rho_{\mathrm{g}}}} \\
& K_{\mathrm{RT}}=\sqrt{\frac{-g_{\mathrm{T}}\left(\rho_{\mathrm{P}}-\rho_{\mathrm{g}}\right)}{3 \sigma} .}
\end{aligned}
$$

The KH breakup time $\tau_{\mathrm{KH}}$ is calculated using Eq. 20. In the case of secondary breakup, the wavelength of the fastestgrowing wave from $\mathrm{RT}$ instabilities is $2 \pi C_{\mathrm{RT}} / K_{\mathrm{RT}}$, from which $C_{\mathrm{RT}}=0.0001$ is a model constant, and it must be compared to the radius of the droplet. If the wavelength is smaller than the droplet diameter, RT waves are assumed to be growing on the droplet surface. The RT breakup time $\tau_{\mathrm{RT}}$ can then be calculated as shown in Eq. 20,

$$
\begin{aligned}
\tau_{\mathrm{br}-\mathrm{KH}} & =\frac{3.726 B_{1} r}{\Omega_{\mathrm{KH}} \Lambda_{\mathrm{KH}}} \\
\tau_{\mathrm{br}-\mathrm{RT}} & =\frac{C_{\tau}}{\Omega_{\mathrm{RT}}} .
\end{aligned}
$$

Finally, the new stable particle radius is calculated by $r_{\mathrm{c}-\mathrm{KH}}$ when the $\mathrm{KH}$ model is taking place or by $r_{\mathrm{c}-\mathrm{RT}}$ when the RT model is in use, both shown in Eq. 21. Equation 15 is also used here to calculate the decrease in the particle radius, regardless of the model applied (KH or RT), 
$r_{\mathrm{c}-\mathrm{KH}}=B_{0} \Lambda_{\mathrm{KH}}$

$r_{\mathrm{c}-\mathrm{RT}}=\frac{\pi C_{\mathrm{RT}}}{K_{\mathrm{RT}}}$.

In Eqs. 20 and 21, $B_{1}=20 / 0.1$ (RANS $\left./ \mathrm{LES}\right), C_{\tau}=1$, and $B_{0}=0.61$ are the model constants.

\subsubsection{Pilch-Erdman secondary breakup model}

In Pilch and Erdman [33], the concept of maximum stable diameter is used to derive a particle breakup model within a spray composed of fragmenting droplets and considering the velocity during their fragmentation process. The authors define a critical Weber number, shown in Eq. 22, until which no breakup takes place. For higher values of $\mathrm{We}_{\mathrm{P}}$, five droplet breakup regimes are distinguished, ranging from the vibrational regime for small values of $\mathrm{We}_{\mathrm{P}}$ until the wave crest stripping regime at high relative gas-droplet velocities. The characteristic breakup time of each regime is summarized in Table 2.

$\mathrm{We}_{\mathrm{C}}=12\left(1+1.077 O h^{1.6}\right)$.

When the characteristic breakup time $\tau$ is known and based on the value of the Weber number, the velocity of the fragmenting droplet can be derived from the Eq. 23, where $U_{\text {slip }}$ is the slip velocity and $B_{1}=0.75 / 0.375$ (RANS/LES) and $B_{2}=0.348 / 0.2274$ (RANS/LES) are the model constants. With this information, the stable radius of the droplet after breakup is given by Eq. 24, where $V_{\mathrm{d} 1}=\left(1-V_{\mathrm{d}} / U_{\mathrm{slip}}\right)^{2}$,

$V_{\mathrm{d}}=U_{\text {slip }} \sqrt{\frac{\rho_{\mathrm{g}}}{\rho_{\mathrm{P}}}}\left(B_{1} \tau+B_{2} \tau^{2}\right)$

$r_{\text {stable }}=\frac{0.5 \mathrm{We}_{\mathrm{C}} \sigma}{V_{\mathrm{d} 1} \rho_{\mathrm{g}} U_{\text {slip }}^{2}}$.

\subsection{Stochastic turbulent dispersion model}

Turbulent eddies in the gas phase flow may interact with the spray droplets, causing a quicker dispersion of the particles, an effect which would not be observed in the case of a laminar flow in the gas phase. Thus, the relative gas-

Table 2 Characteristic breakup time for the Pilch-Erdman secondary breakup model

\begin{tabular}{ll}
\hline Vibrational $(12<\mathrm{We}<18)$ & $\tau=6.0(\mathrm{We}-12)^{-0.25}$ \\
Bag $(18<\mathrm{We}<45)$ & $\tau=2.45(\mathrm{We}-12)^{0.25}$ \\
Bag and stamen $(45<\mathrm{We}<351)$ & $\tau=14.1(\mathrm{We}-12)^{0.25}$ \\
Sheet stripping $(351<\mathrm{We}<2670)$ & $\tau=0.766(\mathrm{We}-12)^{0.25}$ \\
Wave crest stripping $(\mathrm{We}>2670)$ & $\tau=5.5$ \\
\hline
\end{tabular}

droplet or slip velocity would be written as $u_{\text {rel }}=\tilde{u}+u_{\mathrm{p}}^{\prime}-u_{\mathrm{p}}$, where the additional term $u_{\mathrm{p}}^{\prime}$ is a stochastic velocity component that accounts for the dispersion of the particles. The values of $u_{\mathrm{p}}^{\prime}$ are sampled randomly from a Gaussian distribution with a variance $\sigma=$ $\sqrt{2 k / 3}$ ( $k$ is the turbulent kinetic energy of the smaller eddies) and a mean zero, and written as in Eq. 25 proposed by O'Rourke [35],

$$
G\left(u_{\mathrm{p}, \mathrm{i}}^{\prime}\right)=\frac{1}{\sigma \sqrt{2 \pi}} \exp \left(-\frac{u_{\mathrm{p}, \mathrm{i}}^{\prime}}{2 \sigma}\right)^{2} \text {. }
$$

If one wishes not to consider the aforementioned effect, the value of $u_{\mathrm{p}}^{\prime}$ must be set to zero, and the slip velocity would take its original form $\left(u_{\mathrm{rel}}=\tilde{u}-u_{\mathrm{p}}\right)$, where $\tilde{u}$ is the Favrefiltered velocity and $u_{\mathrm{p}}$ is the droplet velocity.

\subsection{Stochastic collision model}

The last model considered in this study is the collision of droplets within the spray cloud. Keeping track of every single particle path line and determining whether if it crosses the path line of other particles would be very computationally demanding. Instead, the most common approach for addressing this phenomenon is the stochastic model offered by O'Rourke [36]. In this model, one simply calculates the probability that two particles in a computational cell will collide, regardless of their direction. For a collision to take place, the center of the smaller droplet must pass within a flat circle centered around the larger particle with an area $\pi\left(r_{1}+r_{2}\right)^{2}$ perpendicular to the trajectory of the smaller droplet, where $r_{1}$ and $r_{2}$ are the radii of the smaller particle and the larger particle, respectively. The collision volume is then defined as the aforementioned area multiplied by the distance traveled by the smaller particle in one time step, i.e., $\pi\left(r_{1}+r_{2}\right)^{2} v_{\text {rel }} \Delta t$, where $v_{\text {rel }}$ is the relative velocity between particles and $\Delta t$ is the particle time step. If the volume of the computational cell is $V$, the probability that the droplets will collide is given by Eq. 26, where $n_{2}$ is the minimum number of particles between the colliding parcels,

$\bar{n}=\frac{n_{2} \pi\left(r_{1}+r_{2}\right)^{2} u_{\mathrm{rel}}}{V}$.

The probability distribution of the actual number of collisions follows a Poisson distribution, as in Eq. 27,

$$
P(n)=\exp \left(-\bar{n} \frac{\bar{n}^{n}}{n !}\right) \text {. }
$$

After one determines whether the two particles collide, the outcome of the collision must also be determined. If the particles collide head on, they will coalesce, but if the collision is oblique, they will bounce. The latter case is also 
referred to as a grazing collision. To determine this, a critical offset relating the collisional Weber number and the relative radii of larger and smaller droplets is calculated, as shown in Eq. 28, where $f$ is a function of $r_{1} / r_{2}$ as defined in Eq. 29,

$$
\begin{aligned}
& b_{\text {crit }}=\left(r_{1}+r_{2}\right) \sqrt{\min \left(1.0, \frac{2.4 f}{W e}\right)}, \\
& f=\left(\frac{r_{1}}{r_{2}}\right)^{3}+2.7\left(\frac{r_{1}}{r_{2}}\right)-2.4\left(\frac{r_{1}}{r_{2}}\right)^{2} .
\end{aligned}
$$

The actual collision parameter $b=\left(r_{1}+r_{2}\right) \sqrt{Y}$ is a function of the random number $Y$, which ranges from 0 to 1 . If $b<b_{\text {crit }}$, the result of the collision is coalescence; otherwise, the result is a grazing collision. In this study, coalescence was neglected; thus, actions would take place only for grazing collision. If this is the case, the new velocities are calculated based on conservation of momentum and kinetic energy, as shown in Eq. 30,

$$
u_{1}^{\prime}=\frac{m_{1} u_{1}+m_{2} u_{2}+m_{2}\left(u_{1}-u_{2}\right)}{m_{1}+m_{2}}\left(\frac{b-b_{\text {crit }}}{r_{1}+r_{2}-b_{\text {crit }}}\right) \text {. }
$$

\section{Numerical setup}

To tackle all of the numerical problems described above, a code based on the sprayFoam solver from the open-source OpenFOAM toolbox, version 3.0.x was employed. Because the main focus here is to investigate the effect of each individual parameter on the solution, there must be an array of simulation cases for each framework selected. For example, as described in the tree structure in Fig. 3, three different grid resolutions $(0.75,0.50$, and $0.25 \mathrm{~mm})$ are used, which gives us three different categories. The dashed

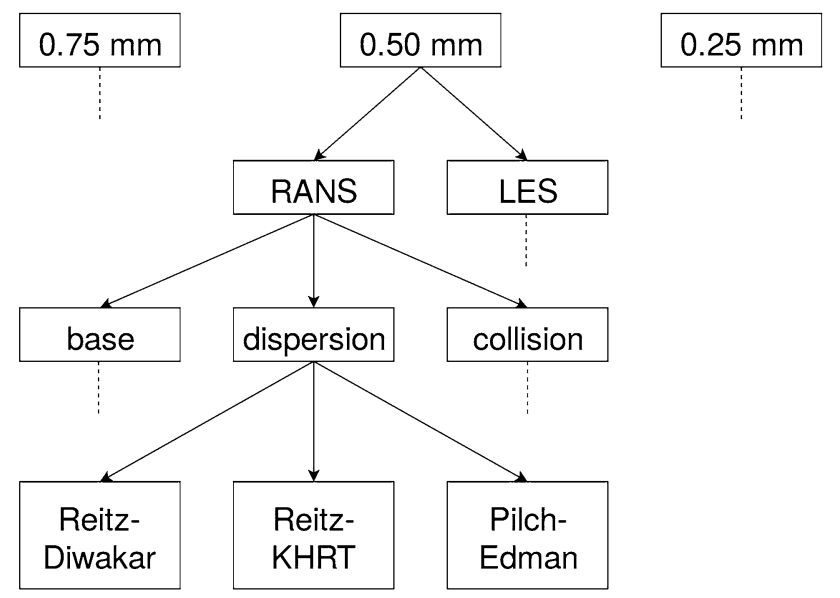

Fig. 3 Structure of simulations line represents the presence of a similar pattern that was not plotted for the sake of simplicity. For each of the resolutions, two turbulence models (RANS $k-\epsilon$ and the standard LES Smagorinsky) will be tested to simulate the turbulence in the gas phase. Furthermore, each different turbulence case will be performed for three flavors of sub-models: the base case, for which no turbulent dispersion and no collision are considered; the dispersion case, in which the additional stochastic turbulent dispersion model is considered; and the collision case, in which the additional stochastic collision model is turned on. Finally, following the branch to the last layer, there are the three distinct secondary breakup models (Reitz-Diwakar, Reitz-KHRT, and Pilch-Erdman). Therefore, $3 \times 2 \times 3 \times 3=54$ simulation cases are needed. The case domain dimensions are the same for all cases $(40 \mathrm{~mm}$ in the radial direction, $30 \mathrm{~mm}$ in the transverse direction, and $60 \mathrm{~mm}$ in the axial direction). A second-order central differencing scheme is used for the solution of the convective terms of the governing equations and an implicit second-order backward scheme is used for the time integration. The standard $k-\epsilon$ model is used in the RANS cases, and for the LES branch, the standard Smagorinsky $\left(C_{\mathrm{s}}=0.168\right)$ is employed to model the subgrid structures. The adjustable time step is set to a maximum CFL of 0.1 , and the simulation total time is set to $2 \mathrm{~ms}$, with an injection duration of $0.78 \mathrm{~ms}$.

\subsection{The Spray G of the engine combustion network}

The simulation case analyzed in this study is known as Spray G of the engine combustion network (ECN), for which the operating conditions are summarized in Table 3. Figure 4 illustrates the spray cloud formed during the injection. Some experimental data are available and were used for the validation of the present model. Because the purpose of this work was to assess the effect of different

Table 3 Spray G [37]

\begin{tabular}{ll}
\hline Fuel & Iso-octane \\
Injection pressure & $20 \mathrm{MPa}$ \\
Fuel temperature & $90{ }^{\circ} \mathrm{C}(363 \mathrm{~K})$ \\
Ambient temperature & $300{ }^{\circ} \mathrm{C}(573 \mathrm{~K})$ \\
Ambient density & $3.5 \mathrm{~kg} / \mathrm{m}^{3}$ \\
Ambient pressure & $6 \mathrm{bar}(600 \mathrm{kPa})$ \\
Ambient composition & $89.71 \% \mathrm{~N}_{2}, 6.52 \% \mathrm{CO}_{2}, 3.77 \% \mathrm{H}_{2} \mathrm{O}$ \\
Injected quantity & $10 \mathrm{mg}$ \\
Injection duration & $780 \mu \mathrm{s}$ \\
Number of nozzle holes & $8($ equally spaced $)$ \\
Hole diameter & $165 \mu \mathrm{m}$ \\
Fully included angle & $80^{\circ}$ \\
\hline
\end{tabular}




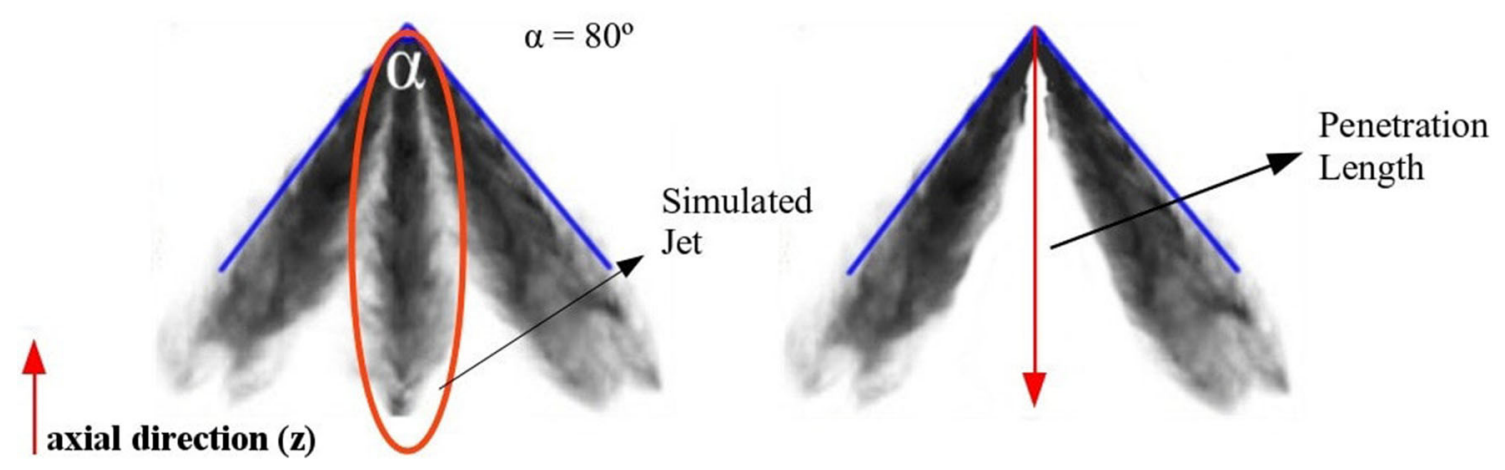

Fig. 4 Spray G [37]. Circled in red, detail of the simulated single jet (color figure online)

parameters on the outcome of important properties of the spray and the gaseous flow, only one jet of eight was simulated. It is important to note that penetration length is measured as a vertical line reflecting the axial direction (z) rather than as the jet axial direction, which is inclined by an angle of $37^{\circ}$ relative to (z) (see Fig. 4 ).

\section{Results and discussion}

\subsection{Spray shape}

In direct injection gasoline engines, the fuel is burned only when it is in the vapor phase; thus, the property of interest for evaluating the quality of the mixture prior to the ignition is the fuel mixture fraction. In these engines, it is important to ensure that an ignitable fuel concentration is present in the vicinity of the spark plug position, from which point the flame will start to propagate. Because the injection of the fuel spray in a gaseous environment exhibits a notably unsteady flow, the shape of the spray cloud in the vapor phase (i.e., the mixture fraction field), is highly non-stationary within the injection duration (in this case, from 0 until $0.78 \mathrm{~ms}$ ).

Vuorinen et al. [12] showed that droplet size may have an important effect on spray shape. To evaluate this effect in the current spray simulations, the Sauter mean diameter (SMD) of the spray droplet cloud was sampled in six different time steps ranging from 0.1 to $0.6 \mathrm{~ms}$. The results of the RANS simulations are presented in Table 4, and the results of the LES simulations are shown in Table 5. Snapshots of the iso-octane mixture fraction field at $0.6 \mathrm{~ms}$ within a plane that cuts the domain down the middle are shown in Figs. 5, 6, and 7, which, respectively, represent the three mesh resolutions used $(0.75,0.50$, and $0.25 \mathrm{~mm})$.

To evaluate the spray penetration length (presented in the next subsection) correctly, two different approaches for the injection were adopted for the LES and RANS simulations that differed in terms of the injection method used and the size of the injected droplets. Although this difference creates an inconsistency between the two turbulence frameworks, it turned out to be necessary, since the influence of the gas phase on the exchange of momentum with the liquid phase differs substantially depending on whether the LES Smagorinsky model or the RANS $k-\epsilon$ model was used. Consequently, good agreement for the penetration length would not be achieved in both turbulence frameworks if the same injection approach had been used. Furthermore, there is substantial uncertainty related to calculations of the flow in the dense spray region close to the nozzle; therefore, such methodology can be justified.

That being said, the size of the droplets (parcels) injected into the computational domain are sampled from a Rosin-Rammler CDF with a characteristic and maximum value equal to $175 \mu \mathrm{m}$ in the RANS simulations and to $120 \mu \mathrm{m}$ in the LES cases. The Blob method is assumed for the generation of the primary droplets, with their velocities being calculated by the Bernoulli relation between nozzle and environment pressures within the RANS simulations, whereas in the LES simulations, the velocities were derived from a table with the fuel mass flow rate and injector geometry properties. Throughout the injection duration, droplet diameter can decrease as a result of only secondary breakup or evaporation. Even when collision is considered, no further change in diameter for any reason other than the aforementioned phenomena can occur, since coalescence is not considered and the outcome of grazing collision is only a change in the velocity of the colliding parcels given by conservation of momentum and kinetic energy.

With that explained, some observations can be made by analyzing the results in Tables 4 and 5. First, relatively small SMDs are obtained relatively quickly after the start of injection in all of the secondary breakup models tested. However, the reduction in size occurs at a considerably faster rate in the RANS/Reitz-KHRT, LES/Reitz-KHRT, and LES/Pilch-Erdman cases. For example, when the 
Table 4 Sauter mean diameter (SMD) $(\mu m)$ of RANS simulations from 0.1 to $0.6 \mathrm{~ms}$

\begin{tabular}{|c|c|c|c|c|c|c|c|c|c|}
\hline \multirow[t]{3}{*}{$t(\mathrm{~ms})$} & \multicolumn{9}{|c|}{ Reitz-Diwakar } \\
\hline & \multicolumn{3}{|c|}{$0.75 \mathrm{~mm}$} & \multicolumn{3}{|c|}{$0.50 \mathrm{~mm}$} & \multicolumn{3}{|c|}{$0.25 \mathrm{~mm}$} \\
\hline & Base & Disp & Col & Base & Disp & Col & Base & Disp & Col \\
\hline 0.1 & 14.13 & 14.1 & 14.43 & 13.75 & 14.19 & 13.83 & 13.36 & 12.79 & 13.20 \\
\hline 0.2 & 7.54 & 7.9 & 8.04 & 7.51 & 7.49 & 8.17 & 7.53 & 7.53 & 7.92 \\
\hline 0.3 & 6.84 & 7.26 & 6.99 & 6.92 & 6.83 & 7.04 & 6.96 & 7.10 & 7.11 \\
\hline 0.4 & 6.39 & 7.32 & 6.98 & 6.48 & 7.28 & 6.94 & 6.98 & 7.52 & 7.31 \\
\hline 0.5 & 6.77 & 7.65 & 6.86 & 6.83 & 7.63 & 7.21 & 6.56 & 7.76 & 7.11 \\
\hline 0.6 & 6.92 & 7.64 & 7.26 & 7.00 & 8.05 & 7.23 & 7.05 & 7.73 & 7.48 \\
\hline \multirow[t]{3}{*}{$t(\mathrm{~ms})$} & \multicolumn{9}{|c|}{ Reitz-KHRT } \\
\hline & \multicolumn{3}{|c|}{$0.75 \mathrm{~mm}$} & \multicolumn{3}{|c|}{$0.50 \mathrm{~mm}$} & \multicolumn{3}{|c|}{$0.25 \mathrm{~mm}$} \\
\hline & Base & Disp & Col & Base & Disp & Col & Base & Disp & Col \\
\hline 0.1 & 1.80 & 1.79 & 1.81 & 1.81 & 1.86 & 1.86 & 2.05 & 2.06 & 2.06 \\
\hline 0.2 & 1.68 & 1.72 & 1.68 & 1.73 & 1.78 & 1.79 & 2.10 & 2.20 & 2.11 \\
\hline 0.3 & 1.61 & 1.65 & 1.61 & 1.67 & 1.71 & 1.72 & 2.12 & 2.13 & 2.19 \\
\hline 0.4 & 1.56 & 1.67 & 1.59 & 1.63 & 1.70 & 1.69 & 2.22 & 2.15 & 2.22 \\
\hline 0.5 & 1.55 & 1.67 & 1.59 & 1.59 & 1.70 & 1.65 & 2.20 & 2.07 & 2.22 \\
\hline 0.6 & 1.55 & 1.66 & 1.59 & 1.55 & 1.61 & 1.62 & 2.15 & 2.00 & 2.22 \\
\hline \multirow[t]{3}{*}{$t(\mathrm{~ms})$} & \multicolumn{9}{|c|}{ Pilch-Erdman } \\
\hline & \multicolumn{3}{|c|}{$0.75 \mathrm{~mm}$} & \multicolumn{3}{|c|}{$0.50 \mathrm{~mm}$} & \multicolumn{3}{|c|}{$0.25 \mathrm{~mm}$} \\
\hline & Base & Disp & $\mathrm{Col}$ & Base & Disp & $\mathrm{Col}$ & Base & Disp & $\mathrm{Col}$ \\
\hline 0.1 & 9.85 & 9.29 & 9.76 & 9.82 & 9.79 & 10.16 & 10.29 & 9.53 & 10.32 \\
\hline 0.2 & 7.39 & 7.75 & 7.72 & 7.51 & 7.99 & 7.90 & 7.85 & 7.85 & 8.19 \\
\hline 0.3 & 6.97 & 7.21 & 7.46 & 7.21 & 7.58 & 7.33 & 7.54 & 7.59 & 8.24 \\
\hline 0.4 & 6.54 & 7.38 & 7.19 & 6.89 & 7.40 & 7.38 & 7.50 & 7.49 & 7.87 \\
\hline 0.5 & 6.63 & 7.50 & 7.06 & 6.99 & 7.81 & 7.14 & 7.27 & 7.53 & 7.69 \\
\hline 0.6 & 6.65 & 7.34 & 7.06 & 6.79 & 7.45 & 7.36 & 7.28 & 7.45 & 7.80 \\
\hline
\end{tabular}

Reitz-Diwakar model is used, the SMD ranges from approximately $13-15 \mu \mathrm{m}$ at $0.1 \mathrm{~ms}$, and from approximately $4-8 \mu \mathrm{m}$ at $0.6 \mathrm{~ms}$. In contrast, the use of the ReitzKHRT model produces an SMD value that ranges from 0.4 to $2 \mu \mathrm{m}$ during the period from 0.1 to $0.6 \mathrm{~ms}$ instants. Furthermore, the reduction in size when the Pilch-Erdman model is used is somewhat similar to that seen in the case of the Reitz-Diwakar model within the RANS framework, but with slightly smaller diameters (approximately $10 \mu \mathrm{m}$ ) at $0.1 \mathrm{~ms}$. However, in the LES framework, the SMDs in the Pilch-Erdman cases are more similar to those obtained in the Reitz-KHRT cases. Thus, the general trend captured herein is that the Reitz-Diwakar model produces much smoother breakup from larger to smaller droplets, whereas the other two models tested tend to produce monodisperse size distributions of considerably smaller values.

An interesting increase in SMD over time was observed in the LES/Pilch-Erdman cases, findings which are in opposition to that which was observed in the other cases: in the other cases, the SMD typically decreased or remained constant over time. This difference may be explained by relatively smaller size reduction rates due to secondary breakup in lower speed breakup regimes. Thus, when the injection process comes closer to the end, the velocity of the injected droplets decrease moderately, and droplet breakup takes place at a more reduced pace. A final note on droplet size is that further turbulent dispersion and collision models do not seem to have a considerable impact on the results, since this trend was observed in almost all mesh resolutions, secondary breakup models, and turbulence frameworks. However, considerable differences were seen in some variations of the LES/Reitz-KHRT and LES/ Pilch-Erdman simulations in terms of those sub-models.

Finally, considerable differences in spray shape were also found depending on whether a RANS or a LES approach was employed. In their modeling of all of the turbulent kinetic energy spectra, the RANS simulations feature fewer turbulent structures than the LES simulations. 
Table 5 Sauter mean diameter (SMD) $(\mu \mathrm{m})$ of LES simulations from 0.1 to $0.6 \mathrm{~ms}$

\begin{tabular}{|c|c|c|c|c|c|c|c|c|c|}
\hline \multirow[t]{3}{*}{$t(\mathrm{~ms})$} & \multicolumn{9}{|c|}{ Reitz-Diwakar } \\
\hline & \multicolumn{3}{|c|}{$0.75 \mathrm{~mm}$} & \multicolumn{3}{|c|}{$0.50 \mathrm{~mm}$} & \multicolumn{3}{|c|}{$0.25 \mathrm{~mm}$} \\
\hline & Base & Disp & $\mathrm{Col}$ & Base & Disp & $\mathrm{Col}$ & Base & Disp & $\mathrm{Col}$ \\
\hline 0.1 & 15.11 & 13.41 & 14.49 & 14.86 & 15.58 & 15.14 & 13.77 & 11.39 & 13.56 \\
\hline 0.2 & 9.41 & 9.25 & 8.63 & 8.77 & 9.60 & 8.38 & 6.19 & 5.36 & 6.91 \\
\hline 0.3 & 7.18 & 6.72 & 6.45 & 6.64 & 6.26 & 6.07 & 5.15 & 4.05 & 5.24 \\
\hline 0.4 & 6.07 & 7.09 & 6.33 & 6.12 & 6.12 & 5.40 & 5.07 & 4.64 & 5.21 \\
\hline 0.5 & 5.97 & 5.34 & 5.39 & 5.44 & 5.11 & 4.79 & 4.29 & 4.15 & 4.58 \\
\hline 0.6 & 4.85 & 4.78 & 4.73 & 4.57 & 4.51 & 4.43 & 4.21 & 3.93 & 4.28 \\
\hline \multirow[t]{3}{*}{$t(\mathrm{~ms})$} & \multicolumn{9}{|c|}{ Reitz-KHRT } \\
\hline & \multicolumn{3}{|c|}{$0.75 \mathrm{~mm}$} & \multicolumn{3}{|c|}{$0.50 \mathrm{~mm}$} & \multicolumn{3}{|c|}{$0.25 \mathrm{~mm}$} \\
\hline & Base & Disp & $\mathrm{Col}$ & Base & Disp & $\mathrm{Col}$ & Base & Disp & Col \\
\hline 0.1 & 0.71 & 0.65 & 0.54 & 0.57 & 0.44 & 0.46 & 0.60 & 1.13 & 1.51 \\
\hline 0.2 & 0.73 & 0.64 & 0.53 & 0.58 & 0.42 & 0.46 & 0.59 & 1.09 & 1.45 \\
\hline 0.3 & 0.72 & 0.65 & 0.53 & 0.57 & 0.43 & 0.46 & 0.60 & 1.09 & 1.40 \\
\hline 0.4 & 0.71 & 0.65 & 0.53 & 0.56 & 0.43 & 0.46 & 0.59 & 1.05 & 1.39 \\
\hline 0.5 & 0.70 & 0.66 & 0.53 & 0.56 & 0.43 & 0.46 & 0.57 & 1.05 & 1.40 \\
\hline 0.6 & 0.70 & 0.66 & 0.53 & 0.55 & 0.44 & 0.46 & 0.58 & 1.05 & 1.41 \\
\hline \multirow[t]{3}{*}{$t(\mathrm{~ms})$} & \multicolumn{9}{|c|}{ Pilch-Erdman } \\
\hline & \multicolumn{3}{|c|}{$0.75 \mathrm{~mm}$} & \multicolumn{3}{|c|}{$0.50 \mathrm{~mm}$} & \multicolumn{3}{|c|}{$0.25 \mathrm{~mm}$} \\
\hline & Base & Disp & $\mathrm{Col}$ & Base & Disp & $\mathrm{Col}$ & Base & Disp & $\mathrm{Col}$ \\
\hline 0.1 & 0.47 & 0.71 & 0.50 & 0.78 & 0.49 & 0.83 & 1.31 & 1.15 & 2.05 \\
\hline 0.2 & 0.55 & 0.77 & 0.50 & 0.82 & 0.47 & 0.87 & 1.36 & 1.07 & 2.20 \\
\hline 0.3 & 0.79 & 0.97 & 0.54 & 0.90 & 0.54 & 0.97 & 1.73 & 1.19 & 2.81 \\
\hline 0.4 & 0.96 & 1.12 & 0.60 & 0.98 & 0.61 & 1.04 & 2.07 & 1.35 & 3.14 \\
\hline 0.5 & 1.13 & 1.24 & 0.63 & 1.03 & 0.65 & 1.11 & 2.12 & 1.42 & 3.22 \\
\hline 0.6 & 1.25 & 1.28 & 0.62 & 1.02 & 0.67 & 1.14 & 2.06 & 1.43 & 3.27 \\
\hline
\end{tabular}

Fig. 5 Iso-octane mixture fraction snapshots at $0.6 \mathrm{~ms}$ after the start of injection for the $0.75 \mathrm{~mm}$ grid. a Base case b dispersion case $\mathbf{c}$ collision case

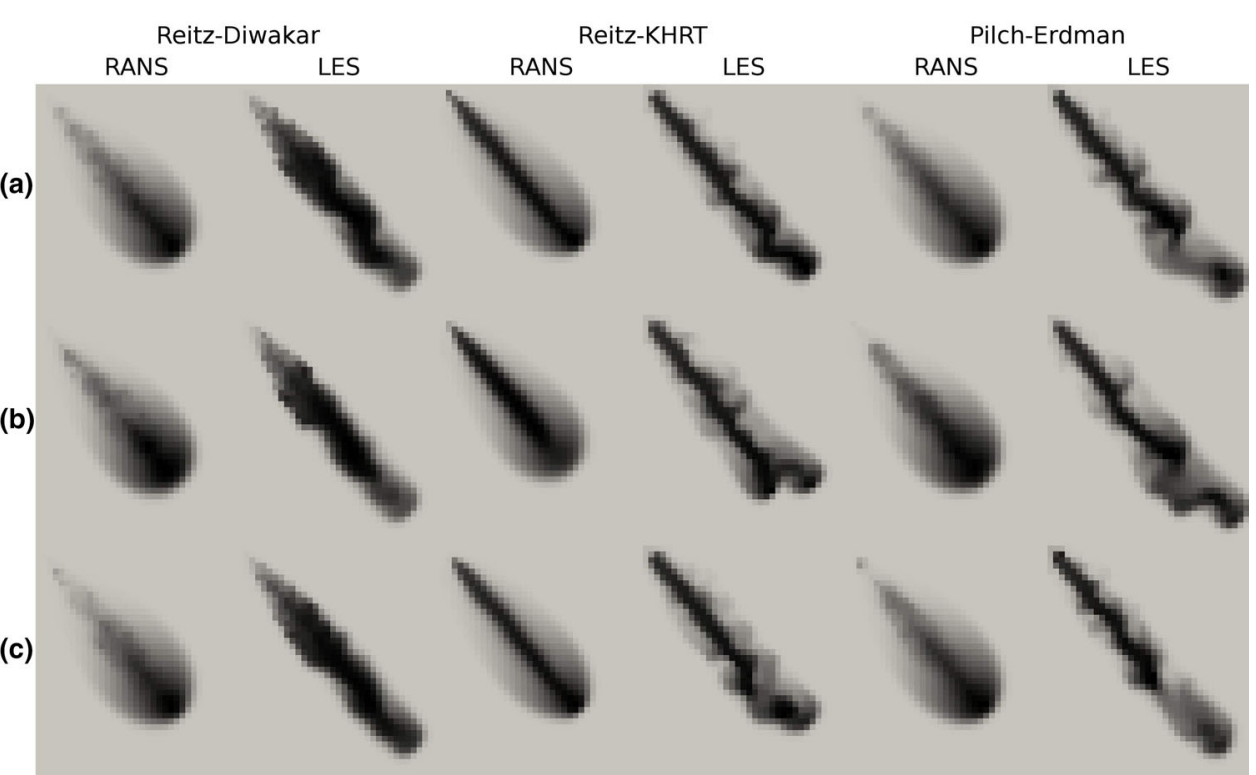


Fig. 6 Iso-octane mixture fraction snapshots at $0.6 \mathrm{~ms}$ after the start of injection for the $0.50 \mathrm{~mm}$ grid. a Base case b dispersion case $\mathbf{c}$ collision case

Fig. 7 Iso-octane mixture fraction snapshots at $0.6 \mathrm{~ms}$ after the start of injection for the $0.25 \mathrm{~mm}$ grid. a Base case b dispersion case c collision case
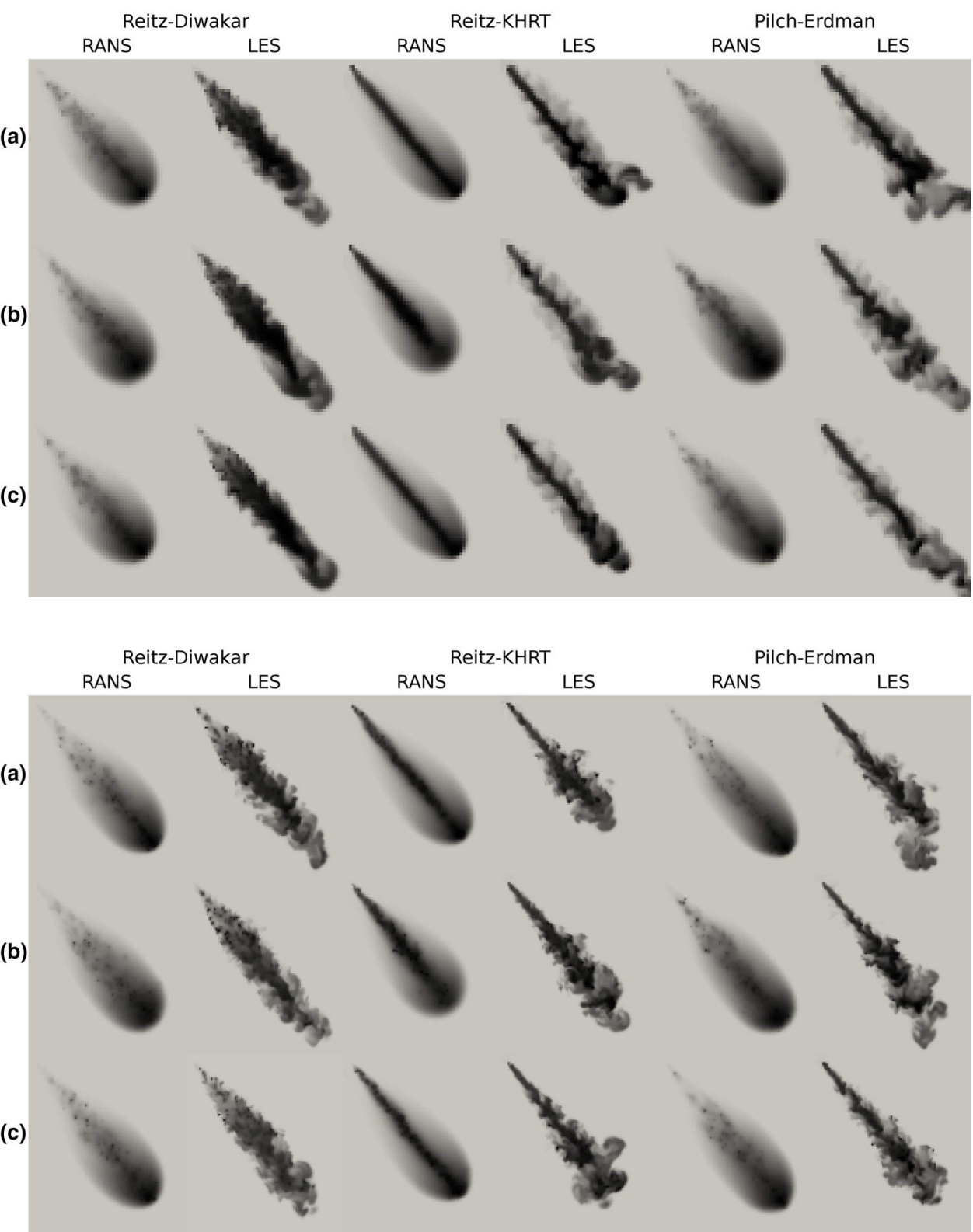

In the RANS framework, virtually no differences were observed between the different mesh resolutions or modeling cases (base, dispersion, and collision), though a slight difference was seen between the Reitz-KHRT cases and the Reitz-Diwakar/Pilch-Erdman cases. Iso-octane mixture fraction values are uniformly concentrated close to the spray's longitudinal axis along injection direction in the former cases, while in the latter cases, higher fraction values were found at the spray tip and increased gradually the farther they were measured from the injection nozzle and the closer they were measured to the spray tip. This behavior in the Reitz-KHRT model is explained by the fast breakup process after the start of injection, which created an uniform distribution of very small droplets in the spray cloud, whereas breakup in the Reitz-Diwakar/Pilch-Erdman cases took place more gradually, generating larger droplets and more dispersion along both the radial and longitudinal axes.

More turbulent structures were seen in the LES simulations, though the mesh seemed to be too coarse at a $0.75 \mathrm{~mm}$ resolution to capture all of them. The higher the resolution, the greater is the number of turbulent structures resolved. Again, further modeling of droplet collision and turbulent dispersion does not seem to have a substantial effect on spray shape, but considerable differences were seen among different secondary breakup models. The spray tip in the simulations with the Pilch-Erdman breakup model appears to have collapsed at the instant the snapshot 


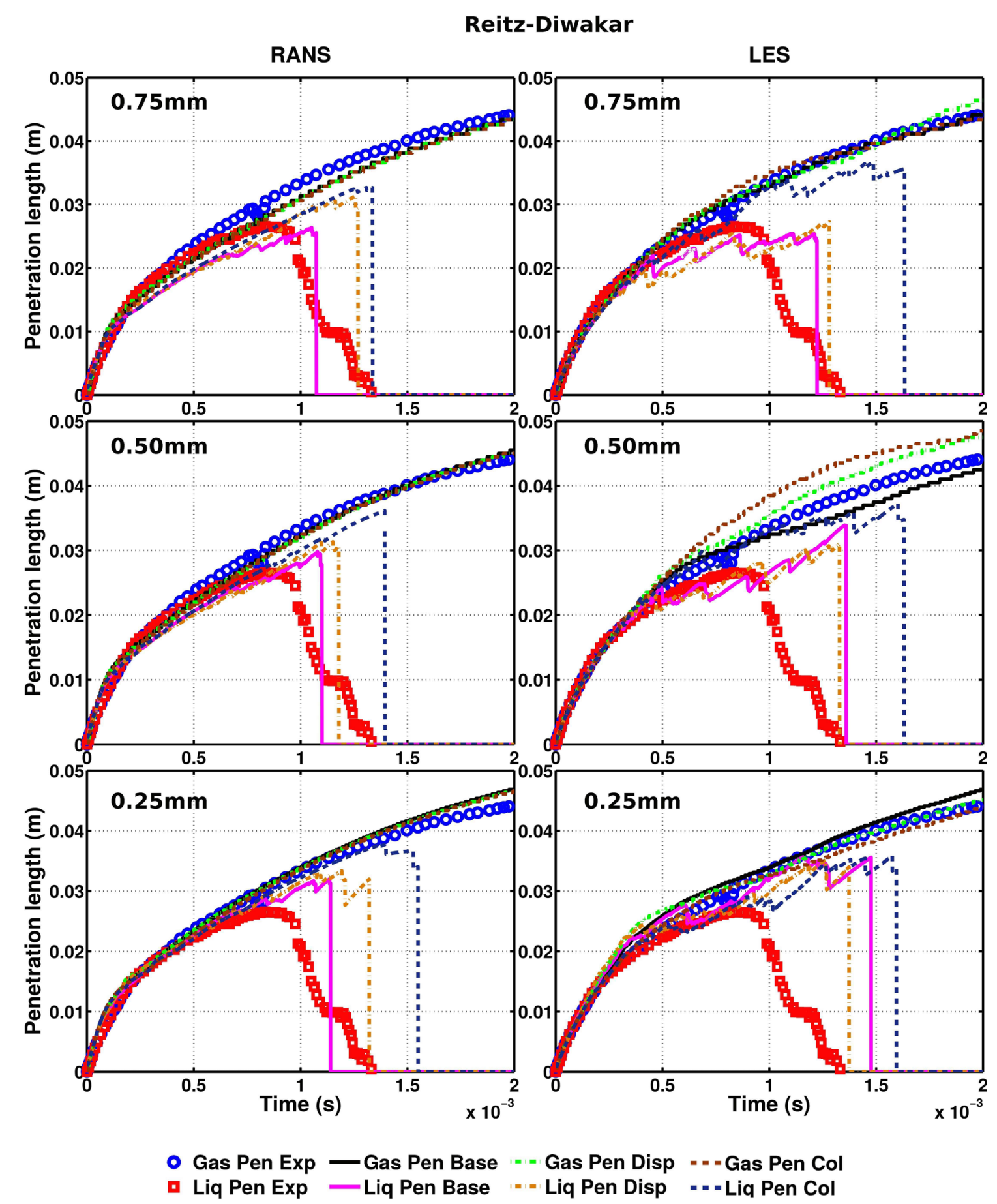

Fig. 8 Spray penetration length in both liquid and vapor phases using the Reitz-Diwakar breakup model. Experimental values have been plotted together for validation

was taken. This behavior can be better observed in the fine $0.25 \mathrm{~mm}$ resolution, which shows some fuel moving away from the spray axis in the radial direction. For coarse and intermediate $(0.50 \mathrm{~mm})$ resolutions, the Reitz-KHRT breakup model creates the same effect seen in the RANS simulations, with high fuel concentrations close to the longitudinal axis. However, this effect is diminished when the resolution is increased to $0.25 \mathrm{~mm}$. Finally, the ReitzDiwakar cases present a more gradual transition from lean to rich conditions, with a higher probability of finding rich mixtures in regions within the spray core. In this case, high concentrations can also be found in some locations at the periphery of the spray cloud, because small droplets that 


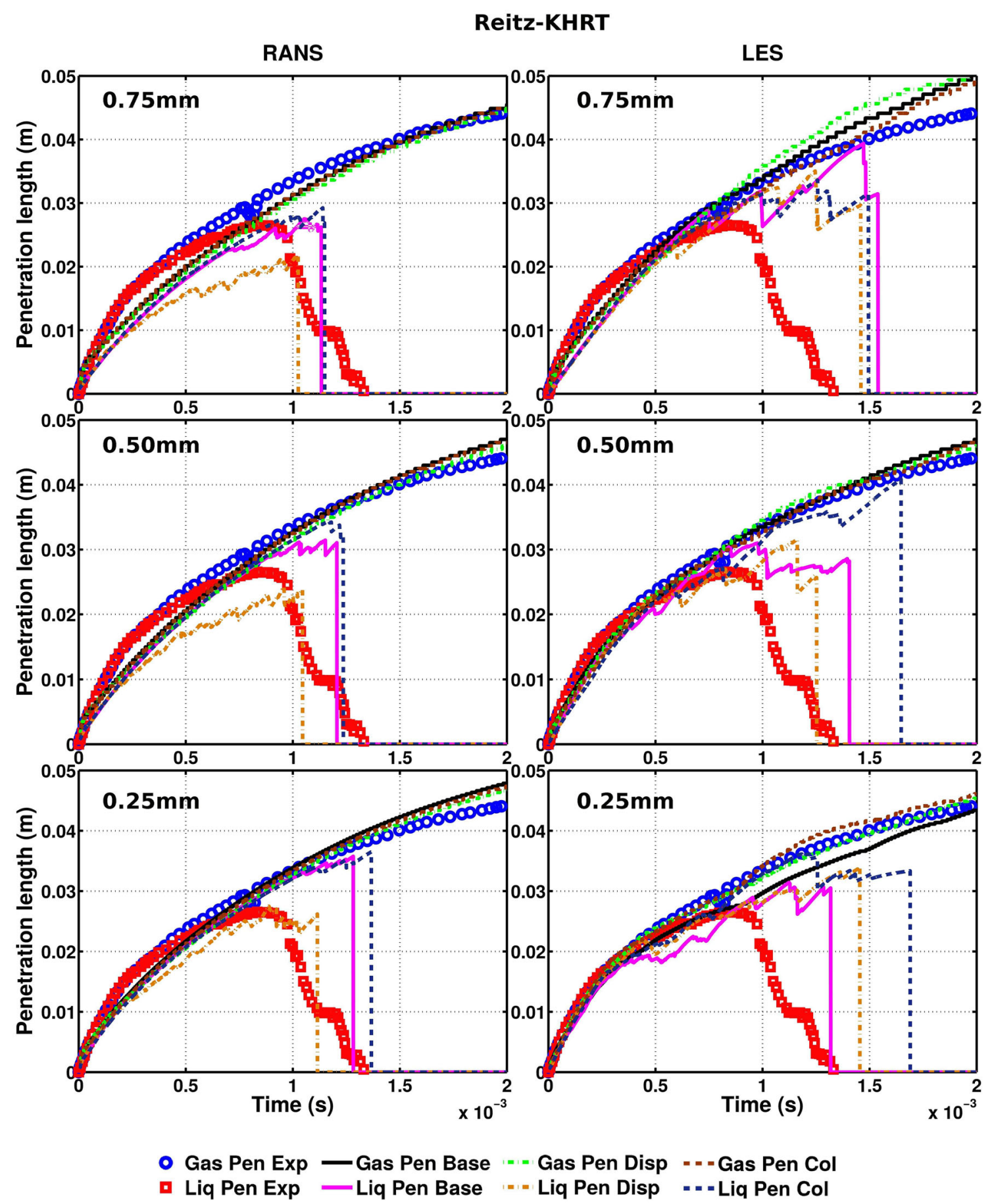

Fig. 9 Spray penetration length in both liquid and vapor phases using the Reitz-KHRT breakup model. Experimental values have been plotted together for validation

evaporate quickly are thrown outside of the spray core due to the strong shear layer.

\subsection{Penetration length}

The second part of this section presents some validation data for the spray penetration length in both the liquid and vapor phases provided from experiments by the ECN [38]. Results for the simulations using the Reitz-Diwakar model are shown in Fig. 8, while results from the Reitz-KHRT are shown in Fig. 9, and those from the Pilch-Erdman model are shown in Fig. 10.

Overall, the simulated vapor penetration length data are very consistent with the experimental results throughout 


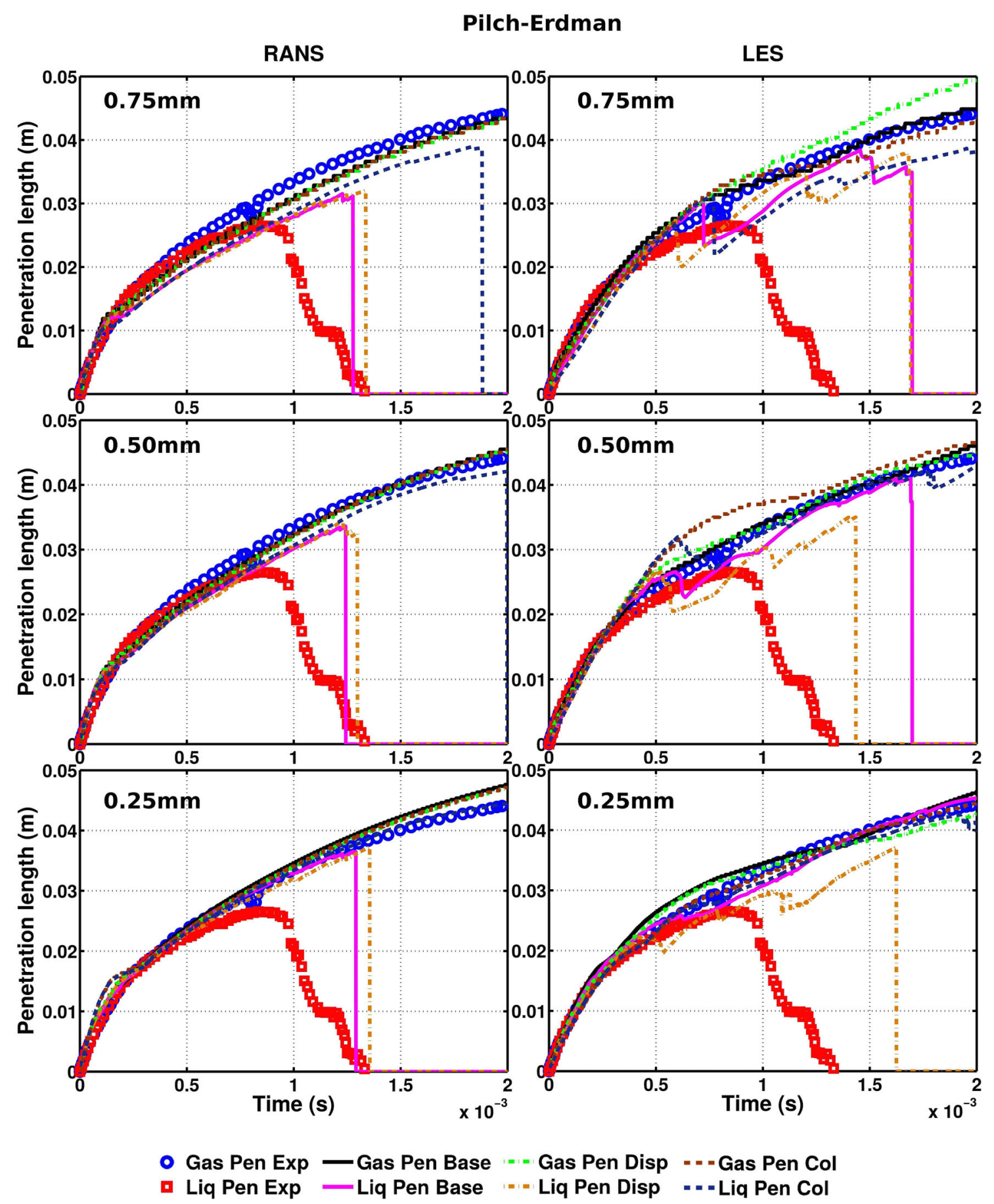

Fig. 10 Spray penetration length in both liquid and vapor phases using the Pilch-Erdman breakup model. Experimental values have been plotted together for validation

the injection duration at all grid resolutions and in both the RANS and the LES simulations. Consistent liquid penetration length was also achieved until the end of injection at $0.78 \mathrm{~ms}$, but its decreasing behavior after that is hard to be resolved with greater accuracy. However, the rough reduction trend until total liquid evaporation was well represented in most cases.
It is also useful to analyze the results separately according to each breakup model. In the case of the ReitzDiwakar model in Fig. 8, spray penetration was found to be somewhat mesh independent in all variations (base, dispersion, and collision) when the RANS $k-\epsilon$ was used. In the LES framework, however, penetration length seemed to be less mesh independent, but good results were obtained 


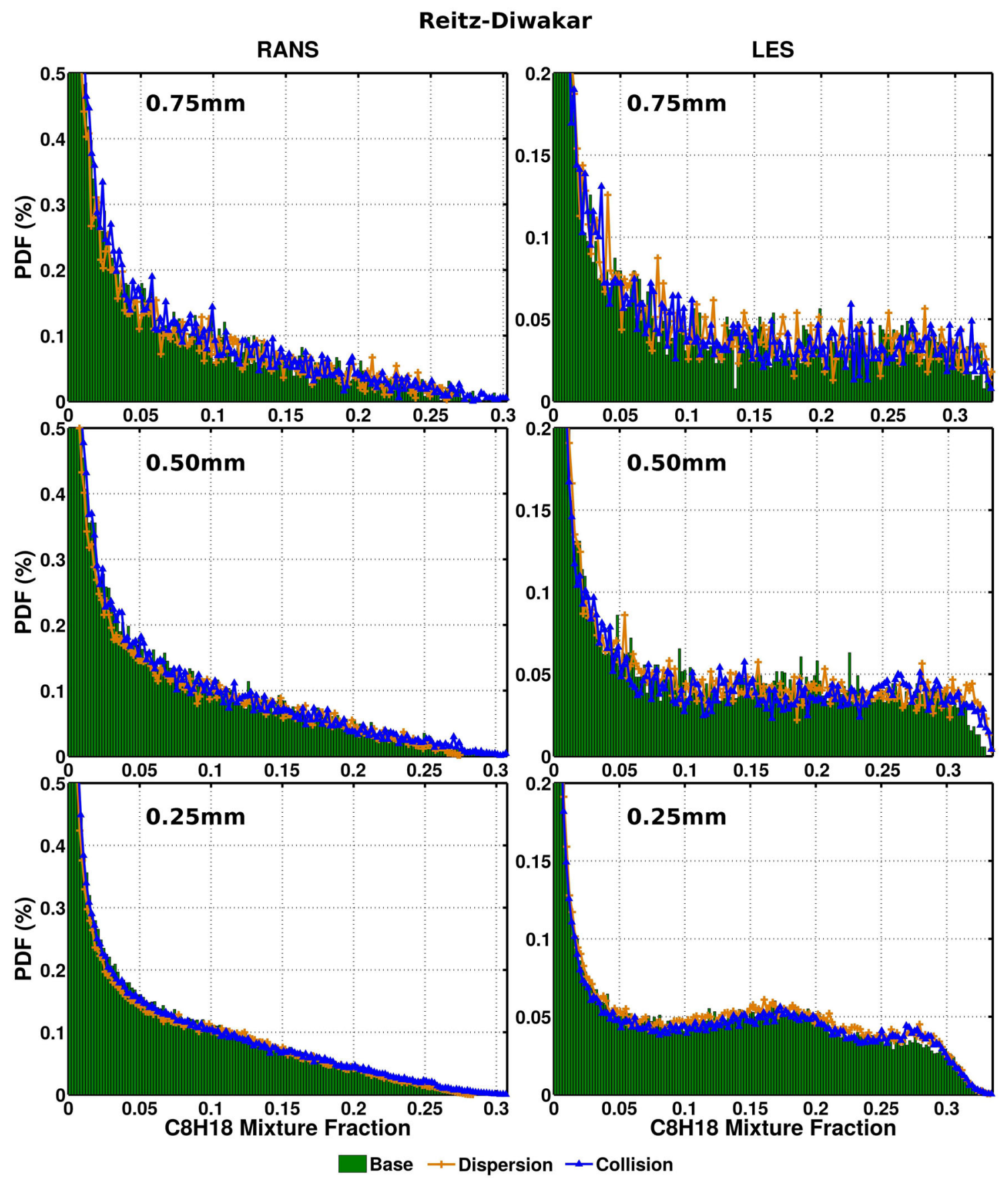

Fig. 11 Probability density function of the iso-octane mixture fraction at $0.6 \mathrm{~ms}$ after the start of injection using the Reitz-Diwakar breakup model and comparing cases: base (green bars), dispersion (yellow line), and collision (blue line) (color figure online)

in both the liquid and the vapor phases, at least in the base case. The consideration of collision and turbulent dispersion induced a trend of overprediction of liquid penetration length in all simulations; for the exceptions were the LES/ $0.50 \mathrm{~mm}$ and LES/ $0.25 \mathrm{~mm}$ simulations, in which the inclusion of dispersion resulted in liquid penetration that was slightly lower than in the base case. The LES/0.50 mm simulations showed the greatest disagreements with experimental values, with the collision/dispersion cases highly overpredicting and the base case slightly underpredicting the vapor penetration length after the end of injection.

In the case of the Reitz-KHRT model in Fig. 9, both liquid and vapor penetration length predictions tended to be underestimated until time instants close to the end of injection. After that point, good agreement was obtained 


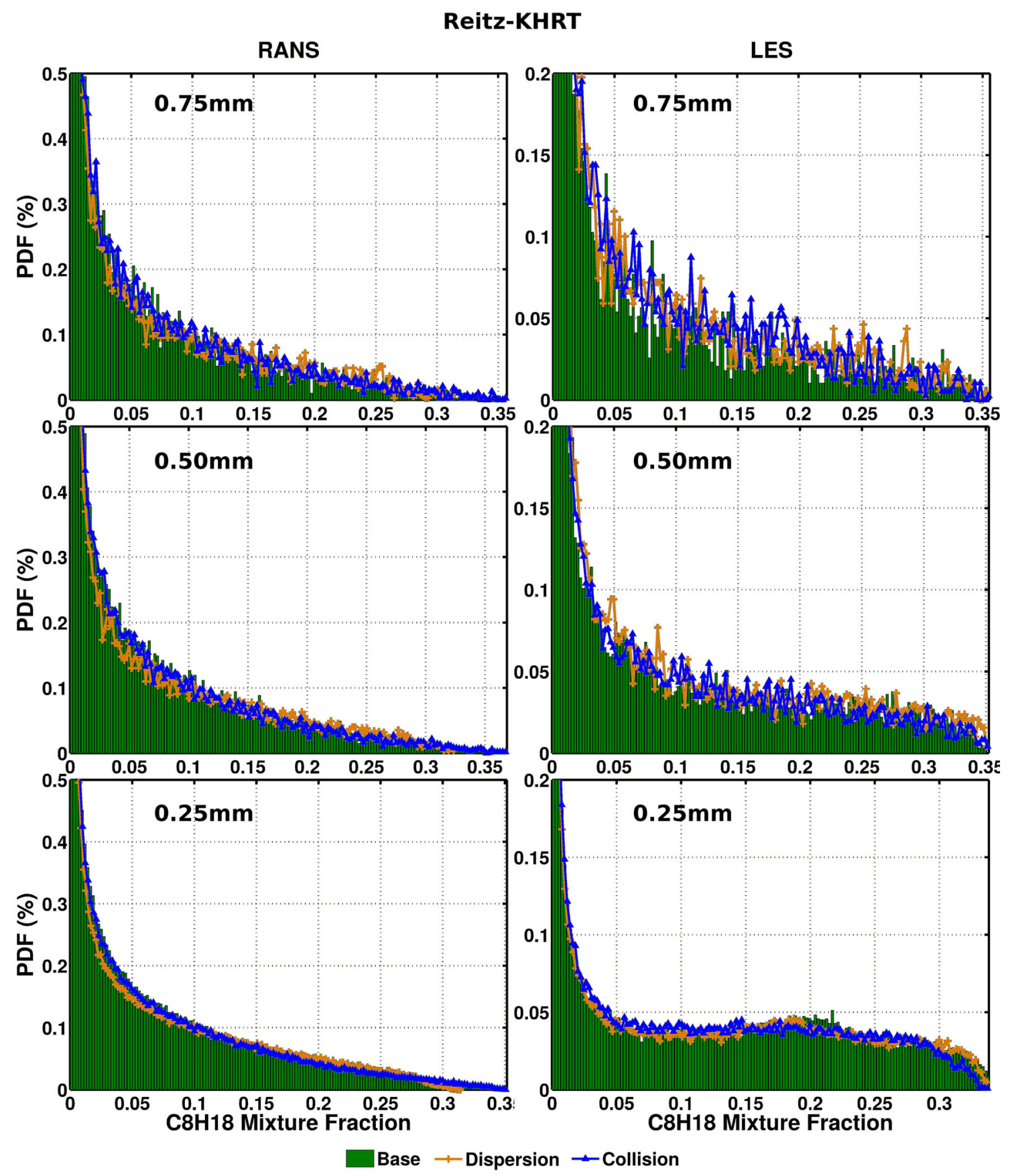

Fig. 12 Probability density function of the iso-octane mixture fraction at $0.6 \mathrm{~ms}$ after the start of injection using the Reitz-KHRT breakup model and comparing cases: base (green bars), dispersion (yellow line), and collision (blue line) (color figure online)

overall in terms of the vapor penetration length and the overall liquid penetration decrease, and liquid vanishing was well represented. In the RANS simulations, the dispersion cases always induced lower liquid penetration, while the inclusion of collision caused a slight increase in liquid penetration. In the LES simulations, the penetration length underprediction until end of injection decreased as the mesh resolution increased. Furthermore, liquid penetration length was found to differ considerably in the base/ dispersion/collision cases, depending on which mesh resolution was used. In the case of coarse mesh, liquid vanishing occurs earlier in the dispersion case, followed by the collision case, then the base case. In the intermediate mesh, total liquid dissipation happens later in the collision case than in the base and dispersion cases. In the case of fine mesh, the same behavior was observed, but the base case presented the lowest values of liquid penetration rather than the dispersion case. Furthermore, the vapor 


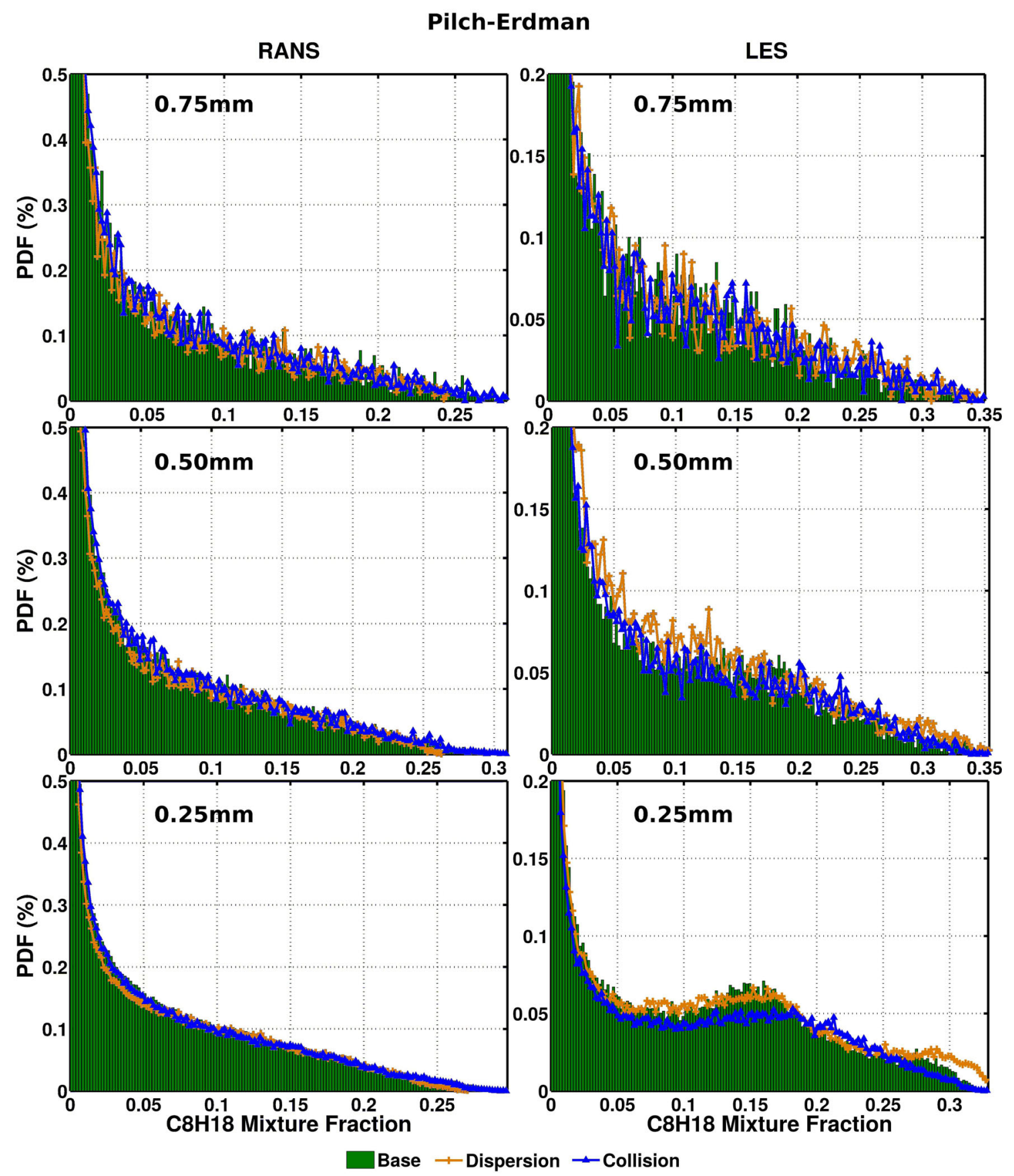

Fig. 13 Probability density function of the iso-octane mixture fraction at $0.6 \mathrm{~ms}$ after the start of injection using the Pilch-Erdman breakup model and comparing cases: base (green bars), dispersion (yellow line), and collision (blue line) (color figure online)

penetration length was found to be considerably underpredicted after the end of injection in the LES/ $0.25 \mathrm{~mm}$ base case.

Finally, in the Pilch-Erdman model in Fig. 10, the results were again found to be slightly grid independent with RANS and considerably grid dependent with LES. All of the cases in which collision was considered presented substantial overprediction of the liquid penetration values.
In the RANS simulations, the inclusion of turbulent dispersion resulted in somewhat decreased liquid penetration but virtually did not affect prediction of the vapor penetration length. In the LES context poor prediction of the liquid penetration length was obtained overall, and overprediction was very high. The vapor penetration predictions, however, were more accurate. 


\subsection{Spray statistics}

The next results to be presented are probability density functions (PDFs) of some important quantities that may have an important effect on the quality of the mixture inside the chamber: the iso-octane mixture fraction at $0.6 \mathrm{~ms}$, which is short before the end of injection at $0.78 \mathrm{~ms}$, and the radial and axial components of the drop and slip velocities, which were sampled from six different time steps ranging from 0.1 to $0.6 \mathrm{~ms}$.

\subsubsection{Iso-octane mixture fraction}

The $\mathrm{C}_{8} \mathrm{H}_{18}$ mixture fraction PDFs of both the RANS and the LES simulations are presented in three different figures. Figure 11 shows the results for the Reitz-Diwakar cases, Fig. 12 shows the results of the Reitz-KHRT cases, and Fig. 13 shows the results of the Pilch-Erdman cases.

The most evident aspect from these results is that no substantial discrepancies were observed between the three modeling variations: the base case, in which no further turbulent dispersion and collision models were considered; the dispersion case, in which only turbulent dispersion was considered; and the collision case, in which only collision was considered. The same trend was observed when the only parameter changed was the grid resolution. However, the turbulence framework and the breakup modeling can affect the results considerably.

For instance, in all variations, the use of the RANS $k-\epsilon$ turbulence model resulted in a higher probability of finding close to zero fuel concentrations than its LES counterpart. Furthermore, the Reitz-KHRT (Fig. 12) cases produced a lower probability of finding rich mixtures and a somewhat higher probability of finding lean conditions than the Reitz-Diwakar model (Fig. 11) cases. This tendency was also found to be slightly higher in the Pilch-Erdman results (Fig. 13).

In addition to the PDFs presented above, we also show the profile of the predicted mixture fraction values averaged over planes along axial direction, as shown in Fig. 14 (for the instant of $0.6 \mathrm{~ms}$ ). Again, the results are greatly influenced by the turbulence modeling strategy used, with the LES model tending to produce a more flattened profile over the axial direction range where mixture fraction values greater than 0 are present, in comparison to the RANS strategy profiles. The RANS profiles have peak values around 0.015 for all grid densities and breakup models, which is somewhat bigger than the peak values seen in the LES profiles that remained below 0.010 in most cases. This behavior agrees with the snapshots previously shown in Figs. 5, 6, and 7, where the RANS snapshots show a clear fuel concentration at the tip of the jet, while the LES
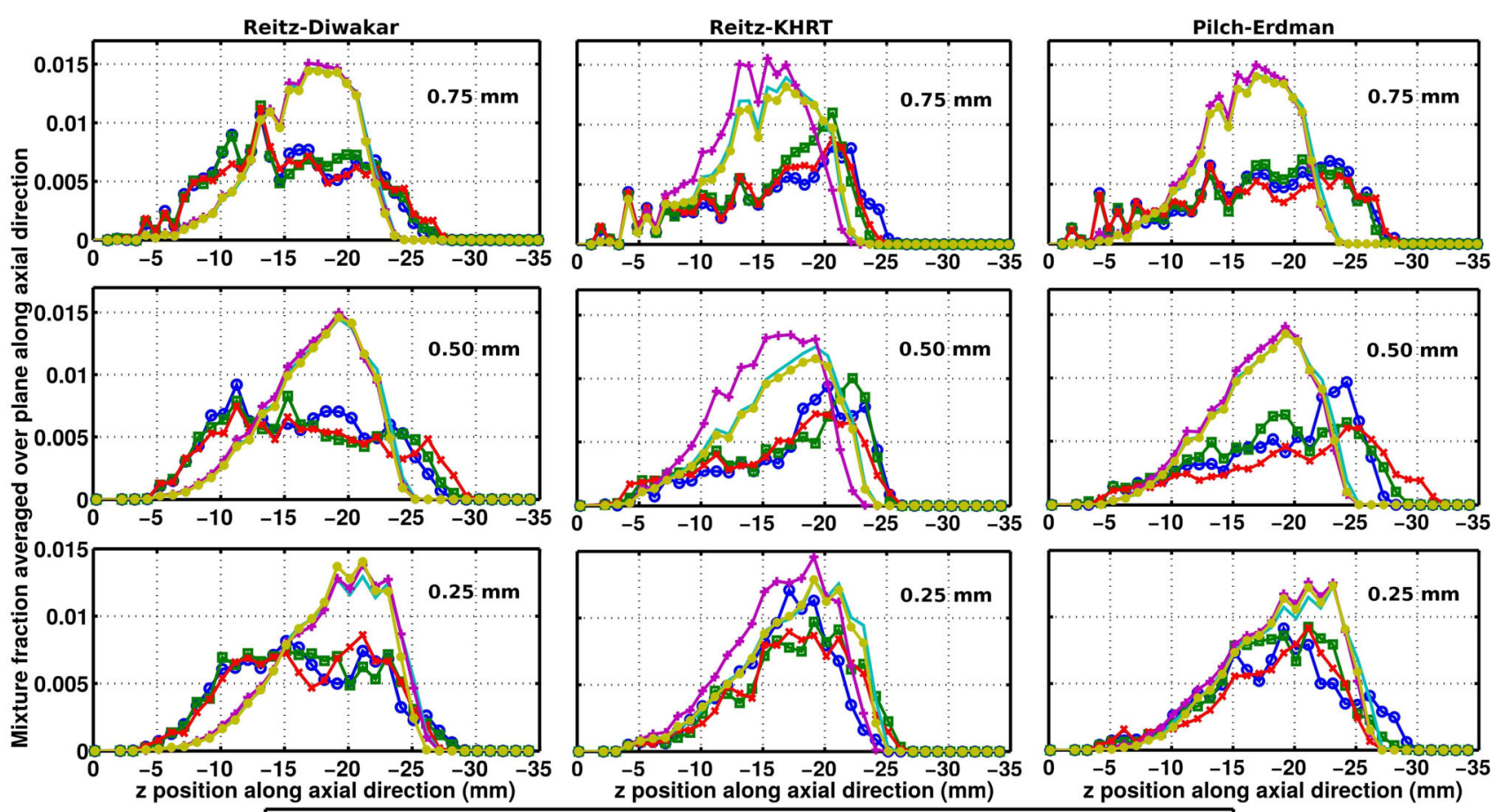

$\rightarrow-$ LES/base $\rightarrow-$ LES/disp $\rightarrow-$ LES/col — RANS/base + RANS/disp $\rightarrow$ RANS/col

Fig. 14 Profiles of averaged fuel mixture fraction at $0.6 \mathrm{~ms}$ after start of injection. Values are averaged over planes at discrete intervals along axial direction (from 0 until $-35 \mathrm{~mm}$ ) 


\section{RANS/Reitz-Diwakar}
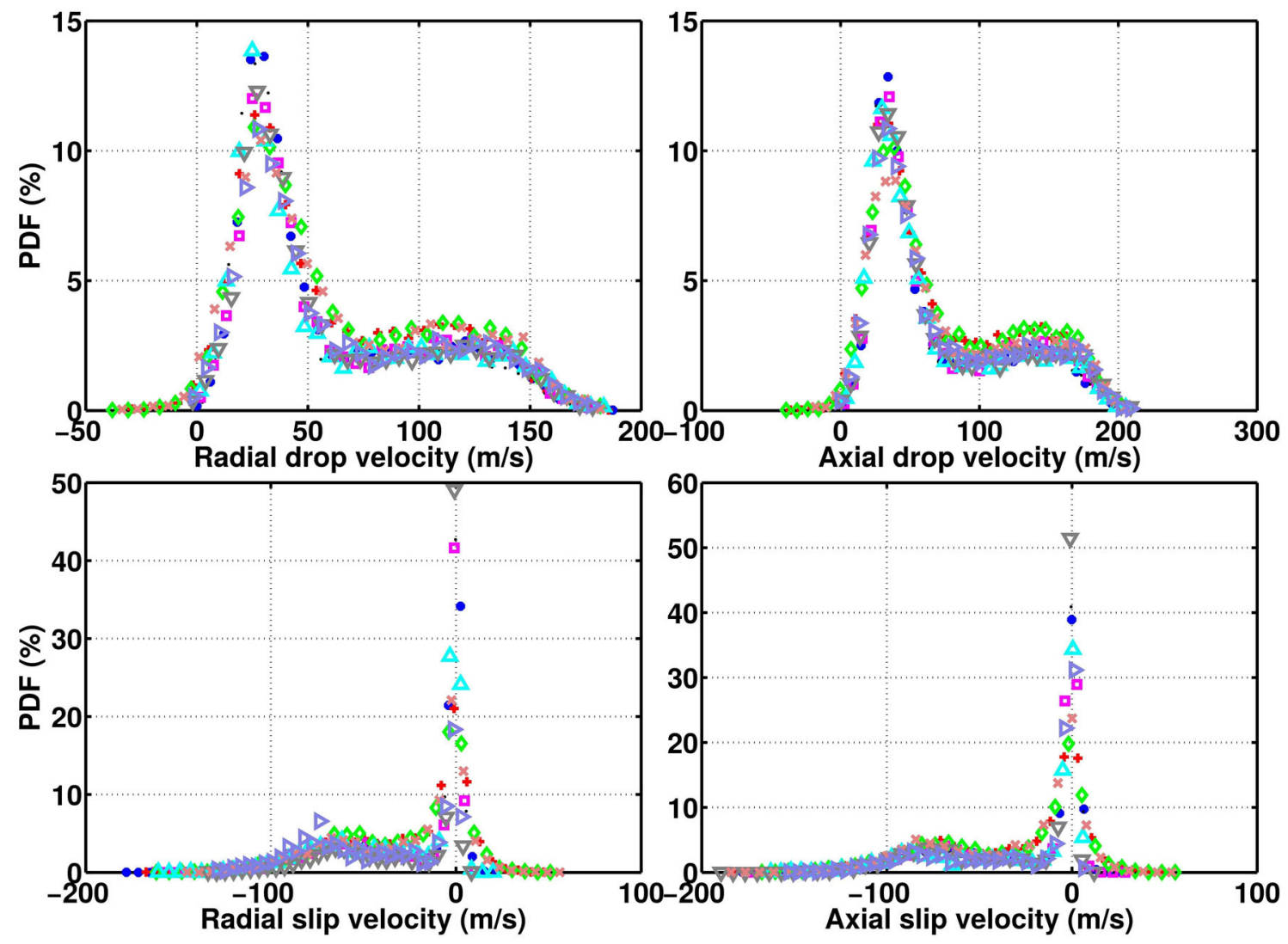

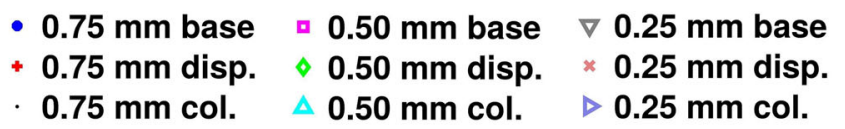

Fig. 15 Probability density function of droplet and slip radial/axial velocities using RANS/Reitz-Diwakar sampled from 0.1 to 0.6 ms

snapshots present a more evenly distributed mass concentration along jet length.

The aforementioned trend is less visible or not observed at all in some LES simulations that use the Reitz-KHRT or the Pilch-Erdman breakup models. For example, for the finest grid density, both Reitz-KHRT and Pilch-Erdman models have LES profiles very similar to the profiles obtained with RANS. Again, this is confirmed by the snapshots, which show that in these particular LES simulations, the tip of the jet tends to have a greater concentration of fuel mass. Finally, holding other variables constant, altering modeling variation, grid density, or breakup modeling, will again have very little effect on these results.

\subsubsection{Flow field}

Two flow field quantities were analyzed. The first flow field considered was drop velocity, which is actually the velocity of the parcel traveling in the chamber, and the second is slip velocity, defined here as $u_{\text {slip }}=u_{\text {gas }}-u_{\text {parcel }}$.

In the RANS/Reitz-Diwakar cases (Fig. 15), the radial droplet PDF was found to peak at approximately $30 \mathrm{~m} / \mathrm{s}$, but velocities as high as approximately $150 \mathrm{~m} / \mathrm{s}$ are likely to be found: they are produced by the high-speed jet exiting the injector nozzle. Somewhat higher velocities were found in the axial direction, since the longitudinal axis of the spray is slightly more inclined in the axial direction than in the radial direction. The slip velocities were also found to form a peak-like curve, but with the highest values centered around $0 \mathrm{~m} / \mathrm{s}$ and with velocities ranging from -170 until $50 \mathrm{~m} / \mathrm{s}$, since the gas is quiescent before the injection takes place.

The LES/Reitz-Diwakar cases (Fig. 16) each present a different M-shape two-peak PDF for both drop and slip velocities. The drop velocity distributions were centered around the values of $50 \mathrm{~m} / \mathrm{s}$ (radial) or slightly higher (axial). In these cases, the maximum velocities were found 


\section{LES/Reitz-Diwakar}
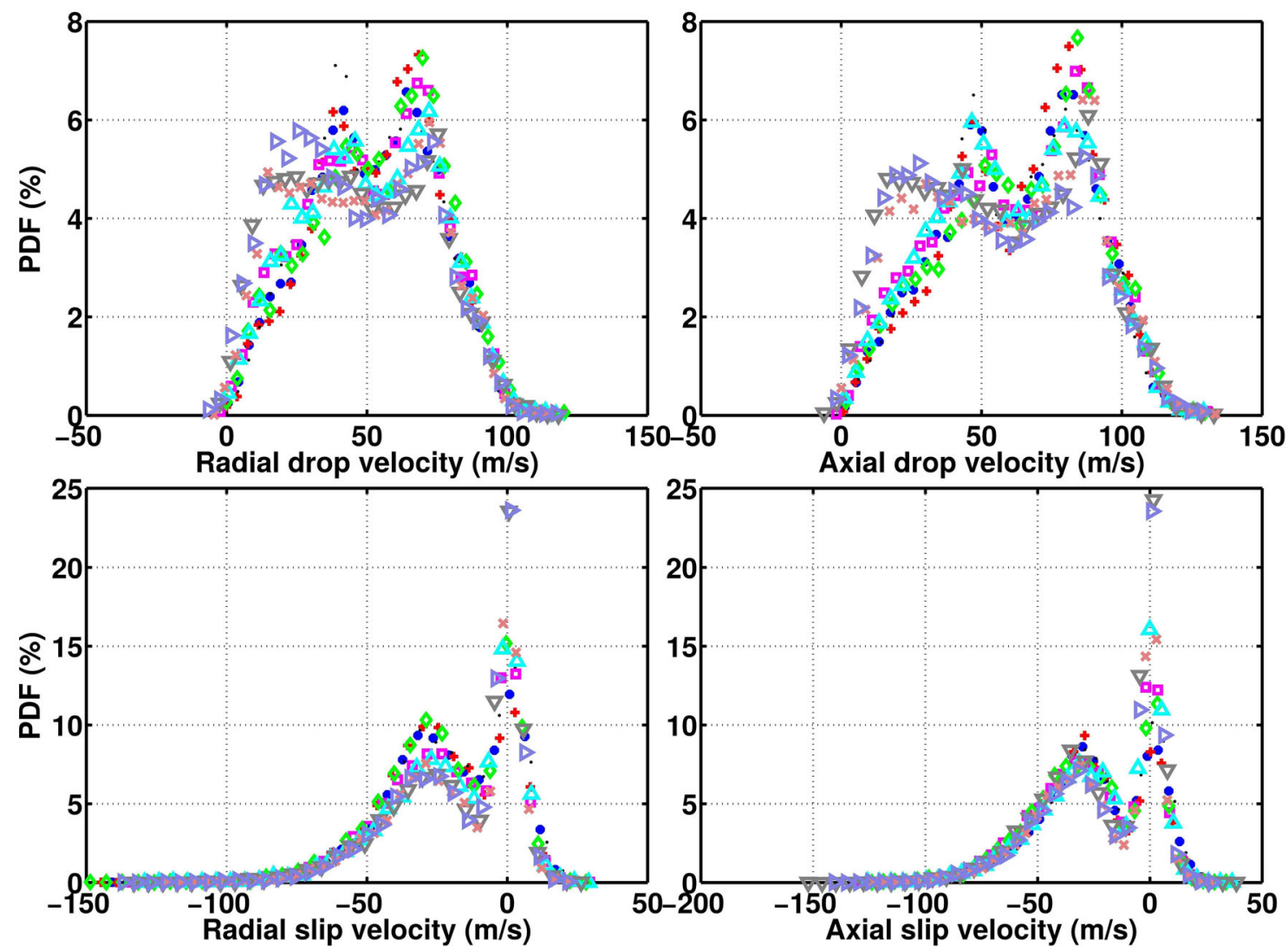

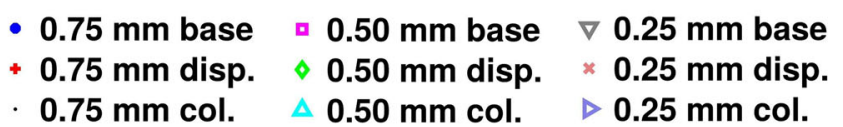

Fig. 16 Probability density function of droplet and slip radial/axial velocities using LES/Reitz-Diwakar sampled from 0.1 to $0.6 \mathrm{~ms}$

to be considerably lower than in the RANS cases. In the case of slip velocity, one peak was found at $0 \mathrm{~m} / \mathrm{s}$ and the other was found at approximately $-30 \mathrm{~m} / \mathrm{s}$. The lower velocities relative to those of the RANS cases are explained by the different injection approaches.

The RANS/Reitz-KHRT cases (Fig. 17) have a one-peak PDF distribution. The peak for the radial and axial drop velocities was found at around $75 \mathrm{~m} / \mathrm{s}$ and was therefore higher than in the peak found in the Reitz-Diwakar cases, but velocities higher than $100 \mathrm{~m} / \mathrm{s}$ are much more likely in these cases than in RANS/Reitz-KHRT cases. Again, the slip velocity PDFs were centered around $0 \mathrm{~m} / \mathrm{s}$, but the distributions were considerably thinner than in the previous breakup model. These thinner velocity distributions were found to be consistent with the droplet size and spray shape results presented in Table 4, since the spray droplet diameters in the Reitz-KHRT model seemed to be more monodisperse.

The LES/Reitz-KHRT cases (Fig. 18) were found to have repeated the trend found in the RANS simulations, though with slightly lower radial and axial drop velocities.
Due to the decrease in droplet velocity, radial and axial slip velocities produced distributions with peaks that were slightly higher than zero. Another trend observed was the fact that the fine mesh caused the radial/axial drop velocity distributions to be more spread out and flatter than those of the other cases.

The RANS/Pilch-Erdman breakup model yielded drop and slip velocity PDFs similar to those obtained in the RANS/Reitz-Diwakar breakup model (Fig. 19). In the LES framework (Fig. 20), however, the distributions were found to be more like those obtained in the Reitz-KHRT breakup model, with a single peak. The collision case, with the 0.50 and $0.25 \mathrm{~mm}$ grids, shifted the peak of axial drop velocity considerably to the right relative to the other variations.

The PDFs presented before give us some insight on statistics of the droplet and slip velocity fields, but not directly of the gaseous phase velocity. So, in the same way as it was done previously for the mixture fraction, Fig. 21 presents profiles of the velocity field in the gaseous phase, averaged over planes along axial direction for the instant of 


\section{RANS/Reitz-KHRT}
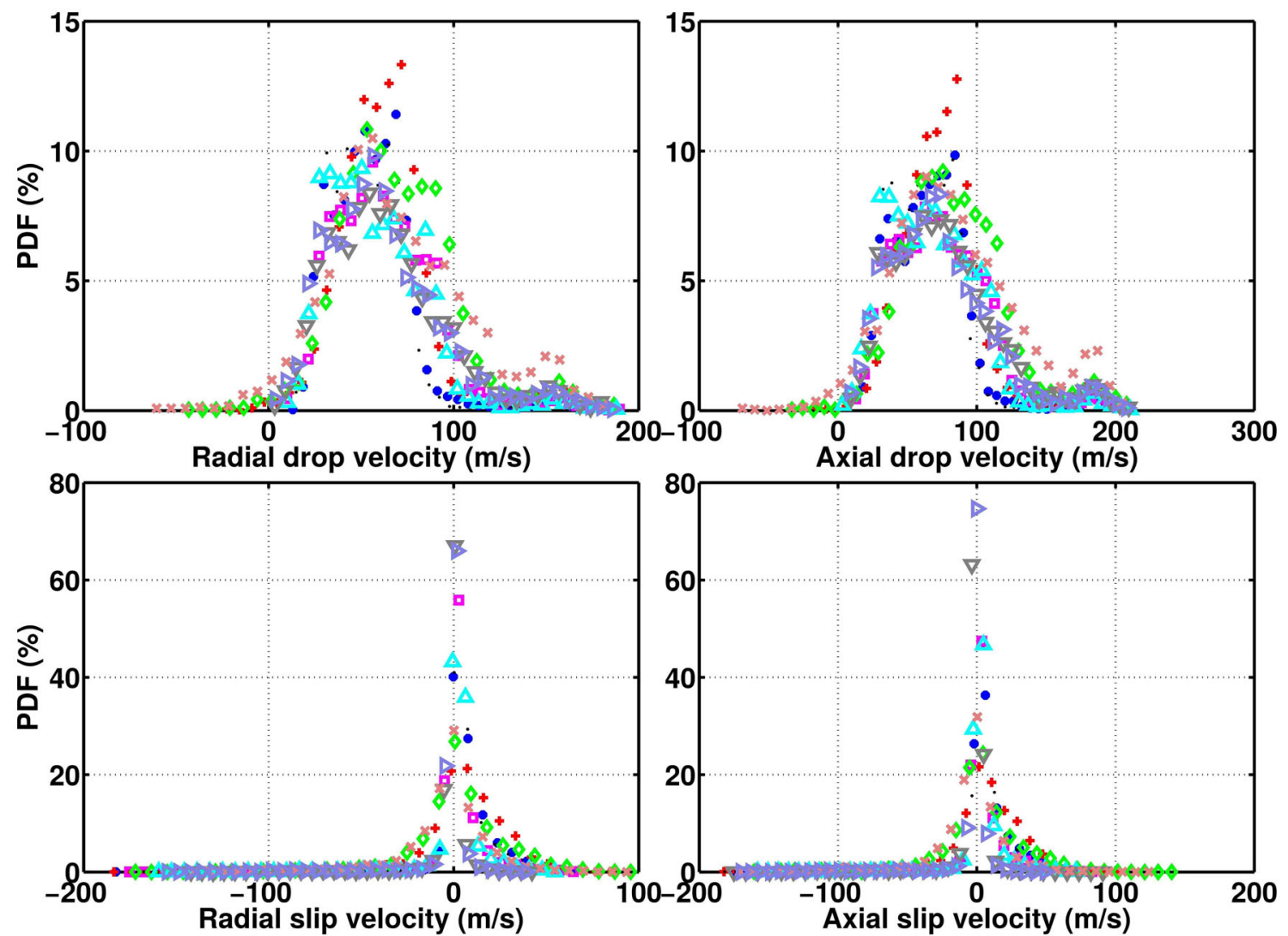

$\begin{array}{lll}\text { - } 0.75 \mathrm{~mm} \text { base } & \square 0.50 \mathrm{~mm} \text { base } & \nabla 0.25 \mathrm{~mm} \text { base } \\ \text { * } 0.75 \mathrm{~mm} \text { disp. } & \nabla 0.50 \mathrm{~mm} \text { disp. } & \star 0.25 \mathrm{~mm} \text { disp. } \\ \text {. } 0.75 \mathrm{~mm} \text { col. } & \Delta 0.50 \mathrm{~mm} \text { col. } & \nabla 0.25 \mathrm{~mm} \text { col. }\end{array}$

Fig. 17 Probability density function of droplet and slip radial/axial velocities using RANS/Reitz-KHRT sampled from 0.1 to $0.6 \mathrm{~ms}$

$0.6 \mathrm{~ms}$. As the gas was quiescent prior to fuel injection, all the profiles tend to show a small deviation from zero. This is due to the fact that only the region within or immediately close to the spray cloud has sufficient time to exchange momentum with the high-speed fuel droplets over the duration of injection. The trend lines of all profiles are very similar to one another, but the RANS profiles tend to present more sharp edges than their LES counterparts. These sharper edges are consistent with the snapshots presented in the spray shape section, which show that when a RANS methodology is employed, the fuel mass is more likely to be found close to spray cloud axis, especially for the Reitz-KHRT breakup model.

\subsection{Comparison between two different LES subgrid models: the standard Smagorinsky model vs. the dynamic k-equation model}

The last contribution of the detailed simulation exercises presented in this article is an investigation of the nature of
LES models for spray simulations. Jet flows present a wide range of turbulent scales, especially within the strong shear flow layer at the boundaries of the spray cloud. The usual approach for LES is to solve the larger scales directly, while the smaller ones are assumed homogeneous and modeled with a set of algebraic equations interconnected by a single constant. However, this homogeneity assumption may not be fair enough and the choice of model constants and/or grid size is key for an accurate simulation. The major drawback of the standard Smagorinsky model, used in the simulations presented so far, is the a priori prescription of a model constant bound to the subgrid stresses that may not be suitable for all flow locations. In our simulations, an implicit filter methodology is employed, which means that not only the LES model constants must be chosen adequately, but the grid resolution must be high enough to represent the characteristics of the flow without significant information loss. Therefore, to assess if the fine mesh resolution used $(0.25 \mathrm{~mm})$ is sufficiently high to resolve most of the turbulent scales present 


\section{LES/Reitz-KHRT}
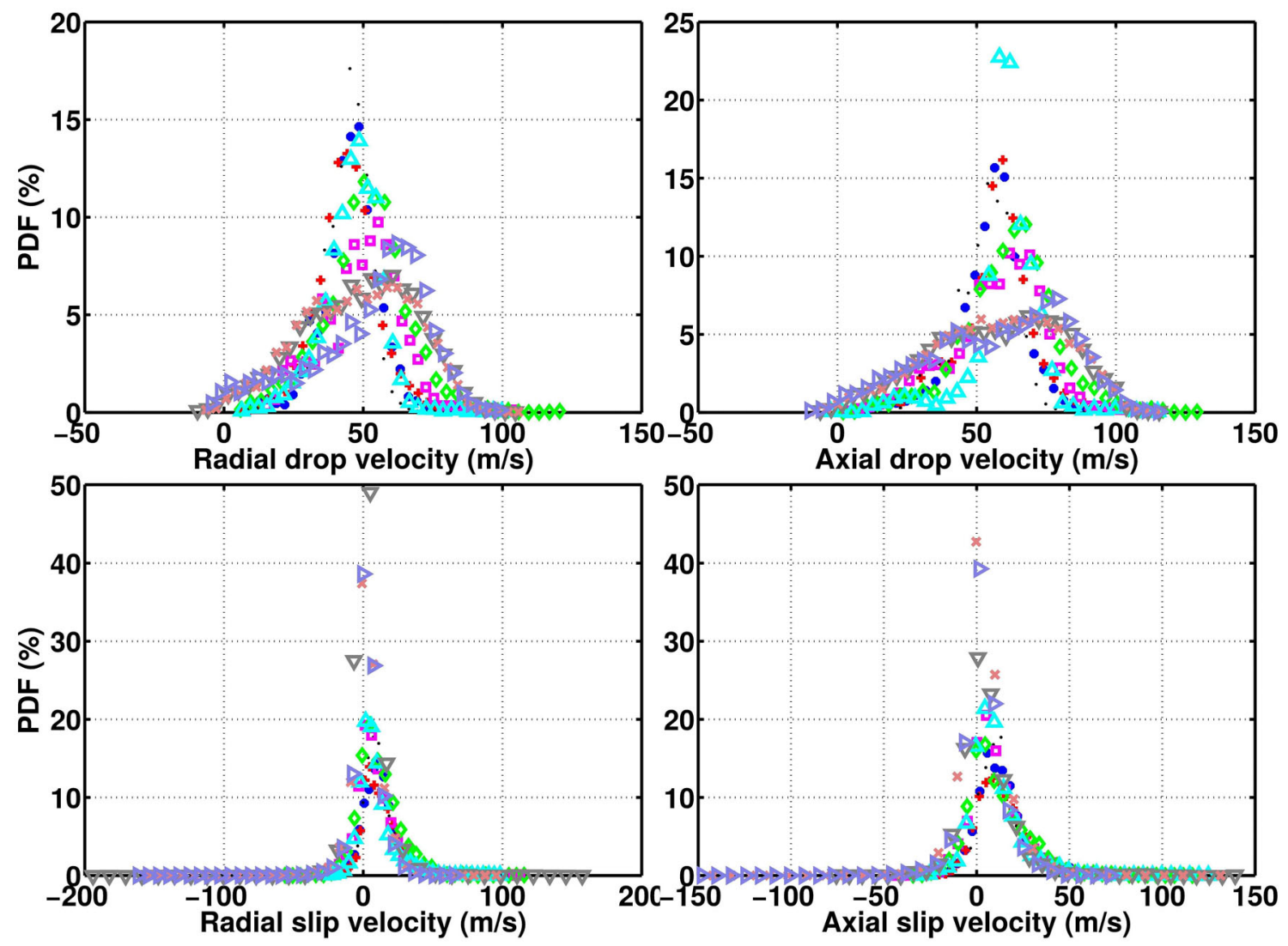

$\begin{array}{lll}\text { - } 0.75 \mathrm{~mm} \text { base } & \square 0.50 \mathrm{~mm} \text { base } & \nabla 0.25 \mathrm{~mm} \text { base } \\ \text { + } 0.75 \mathrm{~mm} \text { disp. } & \diamond 0.50 \mathrm{~mm} \text { disp. } & \star 0.25 \mathrm{~mm} \text { disp. } \\ \text {. } 0.75 \mathrm{~mm} \text { col. } & \Delta 0.50 \mathrm{~mm} \text { col. } & \nabla 0.25 \mathrm{~mm} \text { col. }\end{array}$

Fig. 18 Probability density function of droplet and slip radial/axial velocities using LES/Reitz-KHRT sampled from 0.1 to $0.6 \mathrm{~ms}$

in the studied flow, the results of the base cases are compared to newer simulations using a dynamic subgrid stress modeling approach, which solves an additional transport equation for the turbulent kinetic energy, called dynamic k-equation LES model [39].

In the dynamic k-Eqn model, first an extra transport equation is solved for the subgrid-scale kinetic energy, $k_{\mathrm{sgs}}=1 / 2\left(\bar{u}_{i}^{2}-{\overline{u_{i}}}^{2}\right)$. The subgrid stresses are modeled exactly as before, as $\tau_{i j}=-2 v_{\mathrm{T}} \overline{S_{i j}}+2 / 3 \delta_{i j} k_{\mathrm{sgs}}$, where $\overline{S_{i j}}$ is the resolved scale strain rate tensor. However, the turbulent kinematic viscosity is no longer $v_{\mathrm{T}}=C_{\mathrm{s}}{ }^{2} \overline{\Delta^{2}}|\bar{S}|$ as for the standard Smagorinsky model (where $C_{\mathrm{s}}$ is the Smagorinsky constant), but it is linked to the subgrid kinetic energy instead, as $v_{\mathrm{T}}=C_{v} k_{\mathrm{sgs}}{ }^{1 / 2} \bar{\Delta}$. The one-equation model is then closed by providing a model for the dissipation rate term, $\epsilon=C_{\epsilon} k_{\mathrm{sgs}}{ }^{3 / 2} \bar{\Delta}^{1 / 2}$.

Now, the important feature of this model is that no assumptions of local equilibrium must be made to model the subgrid scales. Instead, the coefficients $C_{v}$ and $C_{\epsilon}$ are calculated using a dynamic process, gathering information from the flow field at every particular time step. Thus, we expect that this model will offer a better prediction of the turbulence. We are not going to discuss the details of this process, since they are out of the scope of this work. However, we highly recommend the work from Kim and Menon for reference [39]. Finally, by comparing the results of this more complex model with the former results using the fine mesh, some evidence can be provided to judge if that resolution is suitable to capture the small scales of turbulence using the simpler standard Smagorinsky LES model.

The methodology for analyzing the results in this section will be exactly the same used so far. First, we compare the SMD of the fuel droplet cloud in six distinct time steps ranging from 0.1 to $0.6 \mathrm{~ms}$, in Table 6 , and then we correlate them with the mixture fraction snapshots obtained for time $0.6 \mathrm{~ms}$ after start of injection, in Fig. 22. After that, plots of liquid/gaseous penetration length are presented in Fig. 23, and mixture fraction PDFs are introduced 


\section{RANS/Pilch-Erdman}
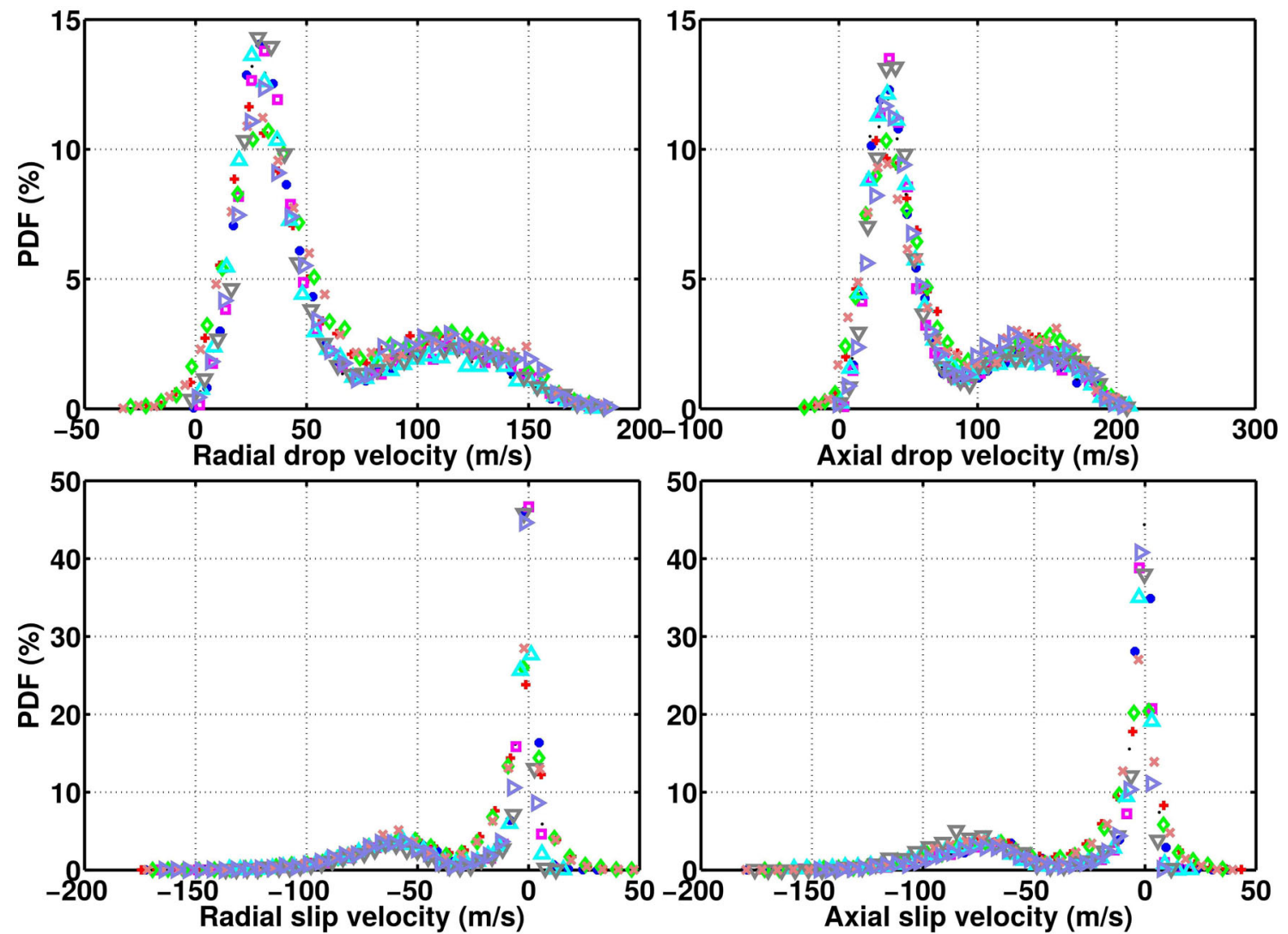

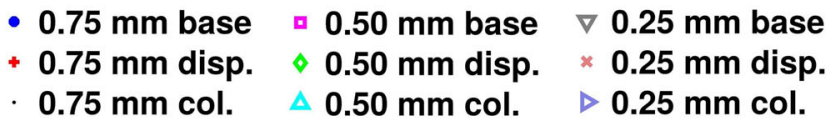

Fig. 19 Probability density function of droplet and slip radial/axial velocities using RANS/Pilch-Erdman sampled from 0.1 to $0.6 \mathrm{~ms}$

in Fig. 24. Furthermore, PDFs of the entire droplet velocity field, as well as the slip velocity field for both LES subgrid models are shown in Fig. 25. Finally, the profiles of the averaged mass fraction and averaged velocity fields are presented in Fig. 26.

Table 6 indicates that for all breakup models but the Pilch-Erdman, the difference in droplet size along time when subgrid models are altered is virtually negligible. For both Reitz-Diwakar and Reitz-KHRT models, the new LES subgrid model decreased the diameter slightly, not more than $10 \%$, while for the Pilch-Erdman model the diameter size increased as high as $20 \%$. This result is coherent with the snapshots portrayed in Fig. 22, which shows no substantial visual difference for the shape of the spray cloud for the different turbulence models together with either the Reitz-Diwakar or the Reitz-KHRT breakup model. However, with the Pilch-Erdman model, the tip of the spray does not seem to collapse when the dynamic $\mathrm{k}$-Eqn subgrid is in use, in opposition to what occurs when the standard Smagorinsky model is used.
Figure 23 shows very little difference between LES subgrid strategies on both liquid and vapor phase penetration length, for all the three breakup models. The only visible disparities are the slightly more delayed liquid vanishing in the simulation with Reitz-KHRT/dynamic $\mathrm{k}$-Eqn models, and the more accurate description of the vapor penetration length before end of injection in the case with Pilch-Erdman/dynamic k-Eqn models, in relation to their counterpart with the standard Smagorinsky model. In Fig. 24, it is also possible to see that no substantial divergence is observed in the distribution of mixture fraction within the domain at $0.6 \mathrm{~ms}$ with the swap of subgrid models, except for an increase in the likeability of finding moderate fuel mass fraction values in the Pilch-Erdman case.

Finally, Fig. 25 shows the comparison of the LES subgrid models in regard to the PDFs of droplet velocity and slip velocity in radial and axial direction, sampled from time instants ranging from 0.1 to $0.6 \mathrm{~ms}$. Furthermore, Fig. 26 presents the comparison in terms of the averaged 


\section{LES/Pilch-Erdman}
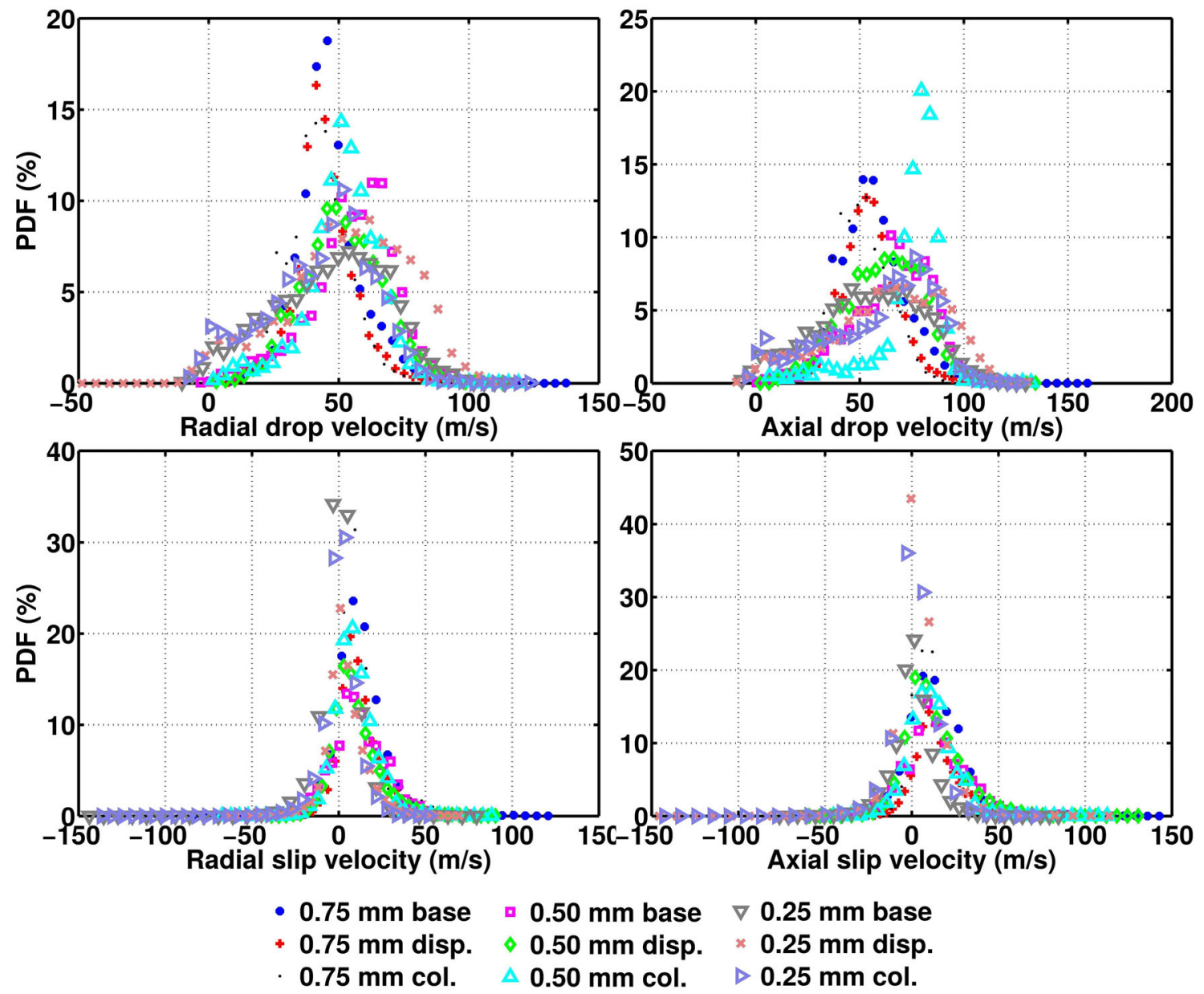

Fig. 20 Probability density function of droplet and slip radial/axial velocities using LES/Pilch-Erdman sampled from 0.1 to $0.6 \mathrm{~ms}$

mixture fraction and the averaged vapor phase axial velocity along axial direction. The values are averaged over planes at discrete intervals along axial direction (from 0 until $-35 \mathrm{~mm}$ ). Again, no big divergences were observed between the two cases, suggesting that the highest resolution used within the LES simulations may be good enough to capture important features of the flow, even with a simpler subgrid model.

\section{Conclusions}

An investigation into the effect of different spray parameters on the outcome of important quantities in the simulation of a single jet from the ECN Spray G condition was performed using a Lagrangian-Eulerian approach and OpenFOAM-3.0.x. These parameters include two different turbulence frameworks (the RANS $k-\epsilon$ and the standard LES Smagorinsky turbulence models), three different mesh resolutions $(0.75,0.50$, and $0.25 \mathrm{~mm})$, three distinct droplet breakup models (Reitz-Diwakar, Reitz-KHRT, and PilchErdman), and further O'Rourke stochastic collision and turbulent dispersion models. Finally, three additional simulations were performed to study specific issues of LES in fuel spray modeling.

The results of this study allow us to conclude that

1. Grid resolution within the range used herein $(0.75-0.25 \mathrm{~mm})$ was found to have virtually no effect on the spray shape in the RANS simulations, but it had significant impacts on the spray shape in the LES simulations. The $0.75-\mathrm{mm}$ grid seemed too coarse for an LES, with very few turbulent structures, while the $0.25-\mathrm{mm}$ grid exhibited a richness in detail compared to the other grids. In more complex simulations, such as the modeling of a complete DISI engine, however, such fine resolution may be hard to achieve; thus, a 0.50 -mm grid may be a compromise between the level of detail and the use of reasonable computational power. 


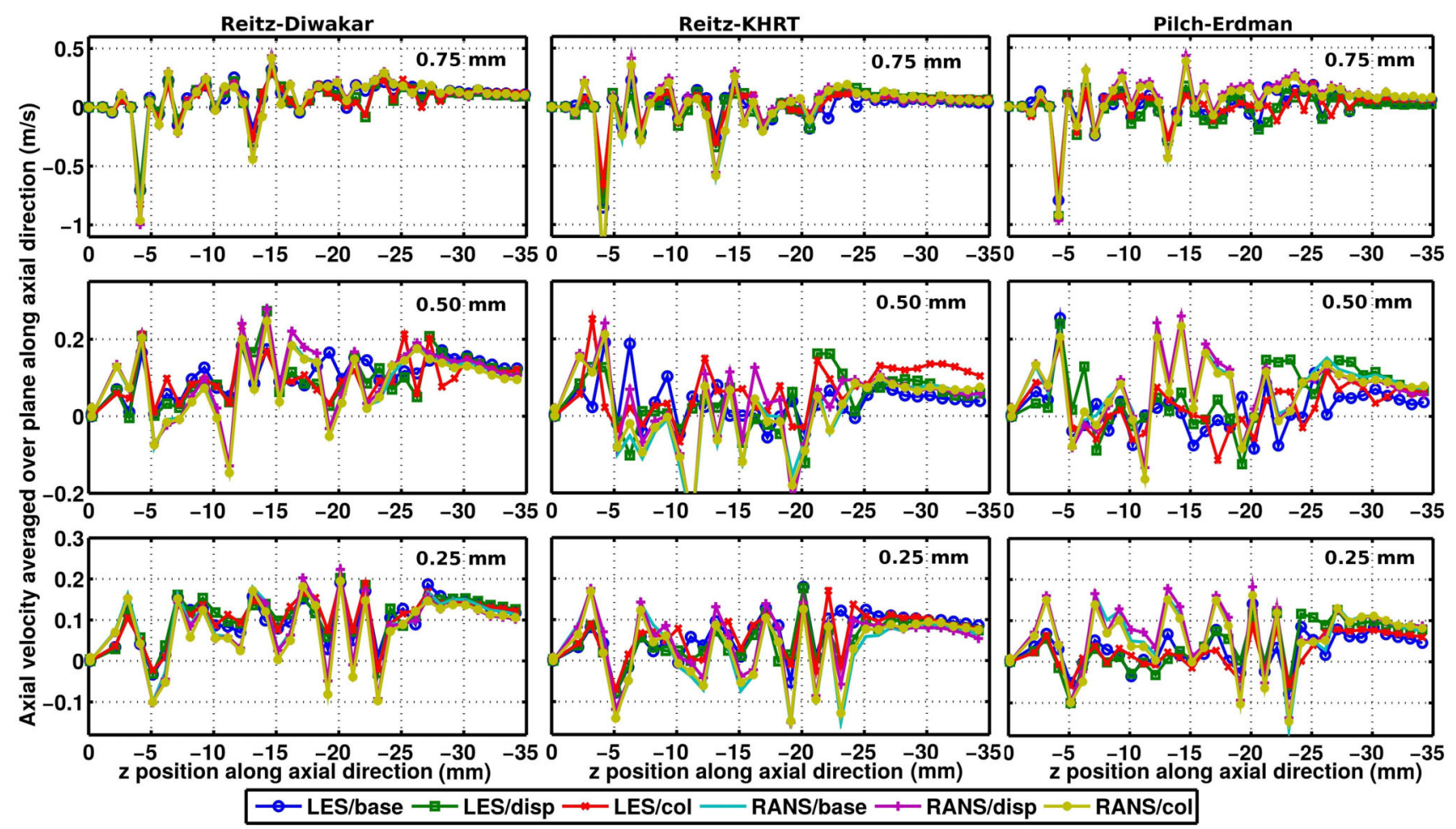

Fig. 21 Profiles of averaged gas phase axial velocity at $0.6 \mathrm{~ms}$ after start of injection. Values are averaged over planes at discrete intervals along axial direction (from 0 to $-35 \mathrm{~mm}$ )

Table 6 Comparison of Sauter mean diameter (SMD) $(\mu \mathrm{m})$ between Std. Smagorinsky and dynamic k-Eqn. simulations

\begin{tabular}{|c|c|c|c|c|c|c|}
\hline \multirow[t]{3}{*}{$t(\mathrm{~ms})$} & \multicolumn{6}{|c|}{ LES comparison between Std. Smag. and Dyn. kEqn. models } \\
\hline & \multicolumn{2}{|c|}{ Reitz-Diwakar } & \multicolumn{2}{|c|}{ Reitz-KHRT } & \multicolumn{2}{|c|}{ Pilch-Erdman } \\
\hline & Std. Smag. & Dyn. kEqn. & Std. Smag. & Dyn. kEqn. & Std. Smag. & Dyn. kEqn. \\
\hline 0.1 & 13.77 & 12.98 & 0.60 & 0.54 & 1.31 & 1.63 \\
\hline 0.2 & 6.19 & 6.16 & 0.59 & 0.54 & 1.36 & 1.64 \\
\hline 0.3 & 5.15 & 4.97 & 0.60 & 0.54 & 1.73 & 2.00 \\
\hline 0.4 & 5.07 & 5.05 & 0.59 & 0.54 & 2.07 & 2.36 \\
\hline 0.5 & 4.29 & 4.44 & 0.57 & 0.53 & 2.12 & 2.40 \\
\hline 0.6 & 4.21 & 4.00 & 0.58 & 0.55 & 2.06 & 2.51 \\
\hline
\end{tabular}

Reitz-Diwakar

Reitz-KHRT

Pilch-Erdman

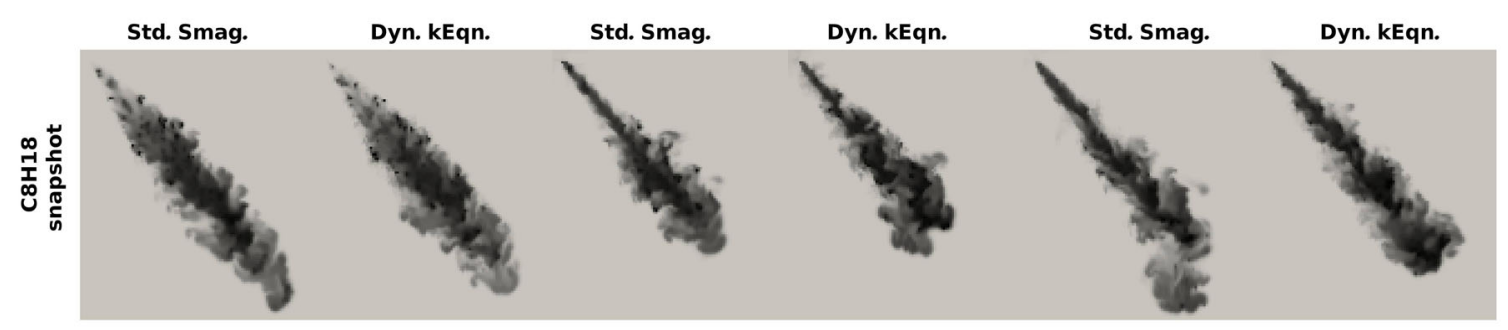

Fig. 22 Iso-octane mixture fraction snapshots at $0.6 \mathrm{~ms}$ after the start of injection

2. The decrease in droplet diameter was much smoother in the RANS/Reitz-Diwakar, LES/Reitz-Diwakar, and RANS/Pilch-Erdman cases, with a significantly more gradual transition from the large injected droplets to the smallest ones. Meanwhile, in the other cases, the reduction in size occurred much more rapidly and 


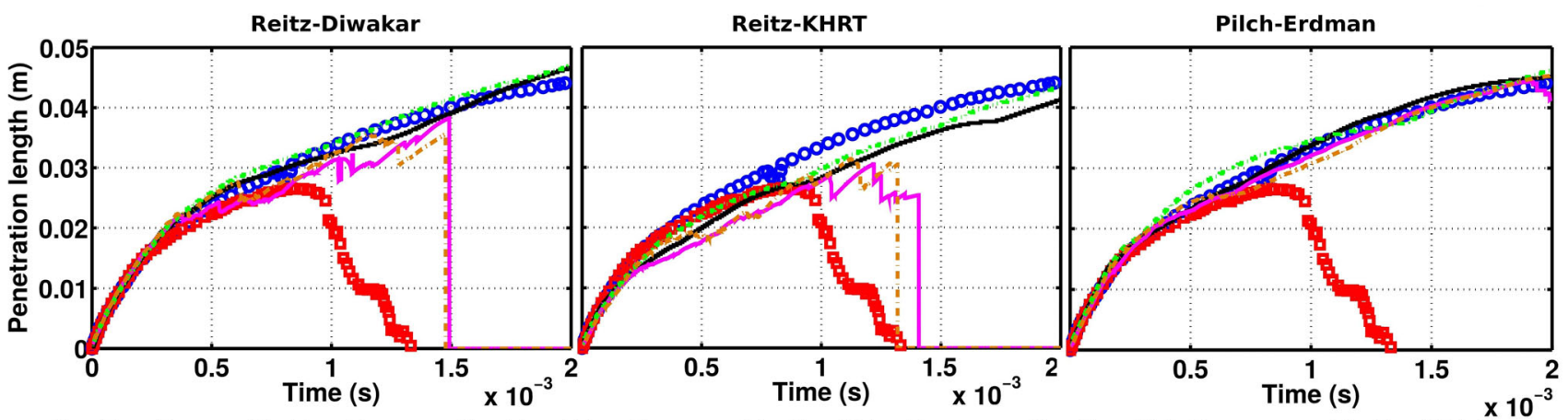

- Gas Pen Exp 0 Liq Pen Exp —Gas Pen kEqn Dyn. — Liq Pen kEqn Dyn. - - Gas Pen Std. Smag. - - Liq Pen Std. Smag.

Fig. 23 Spray penetration length in both liquid and vapor phases. Experimental values have been plotted together for validation
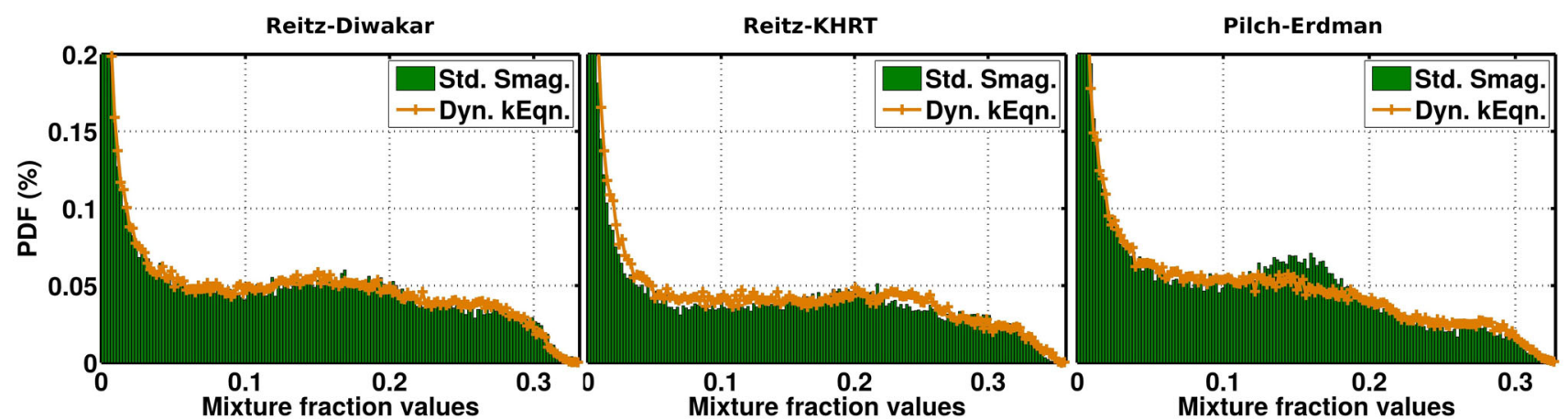

Fig. 24 Probability density function of the iso-octane mixture fraction at $0.6 \mathrm{~ms}$ after the start of injection
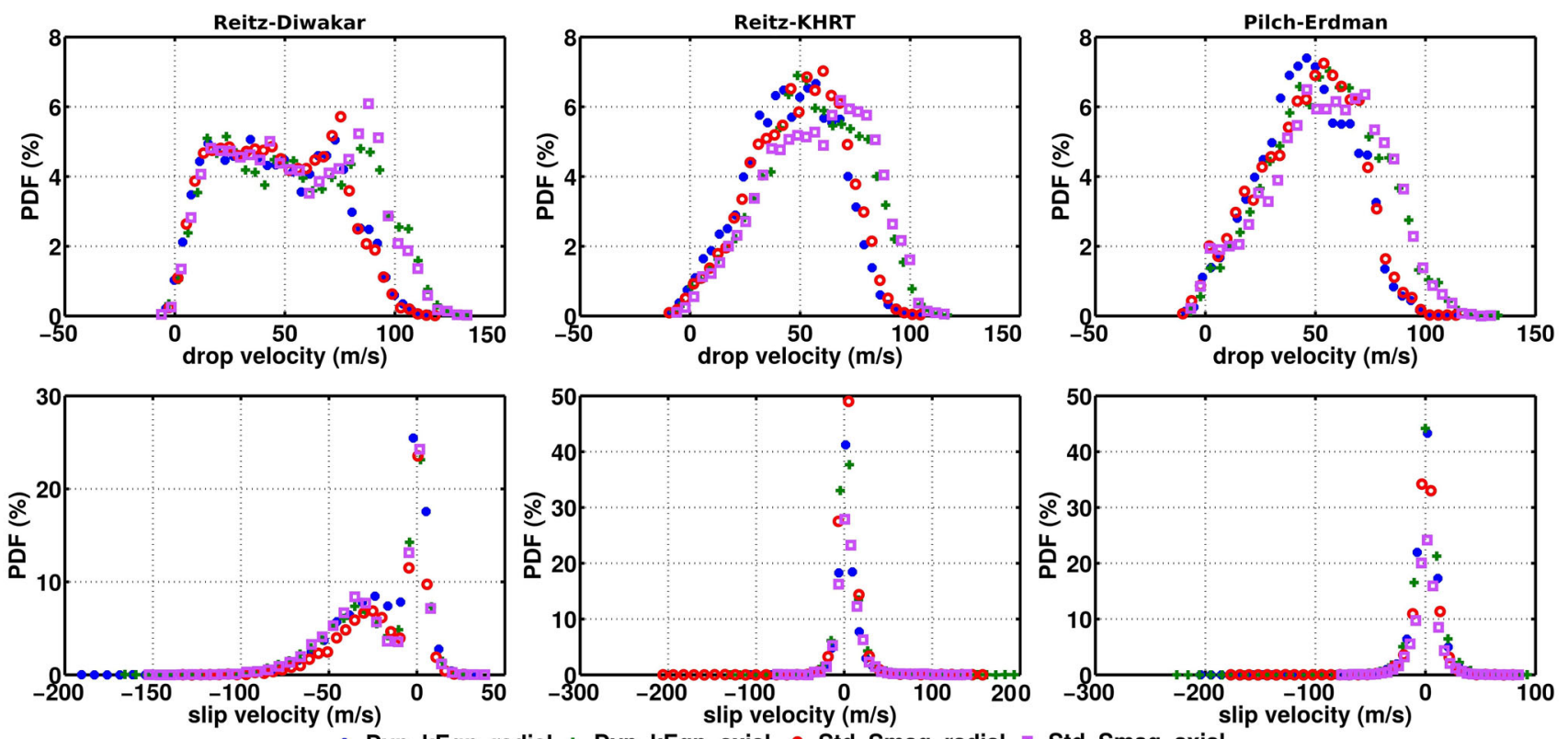

Fig. 25 Probability density function of droplet and slip radial/axial velocities sampled from 0.1 to $0.6 \mathrm{~ms}$

tended to produce a spray with a considerably smaller and monodisperse size distribution.
3. Overall, the simulation results were consistent with the experimental results. In the case of vapor penetration length, the values were consistent until the end of the 

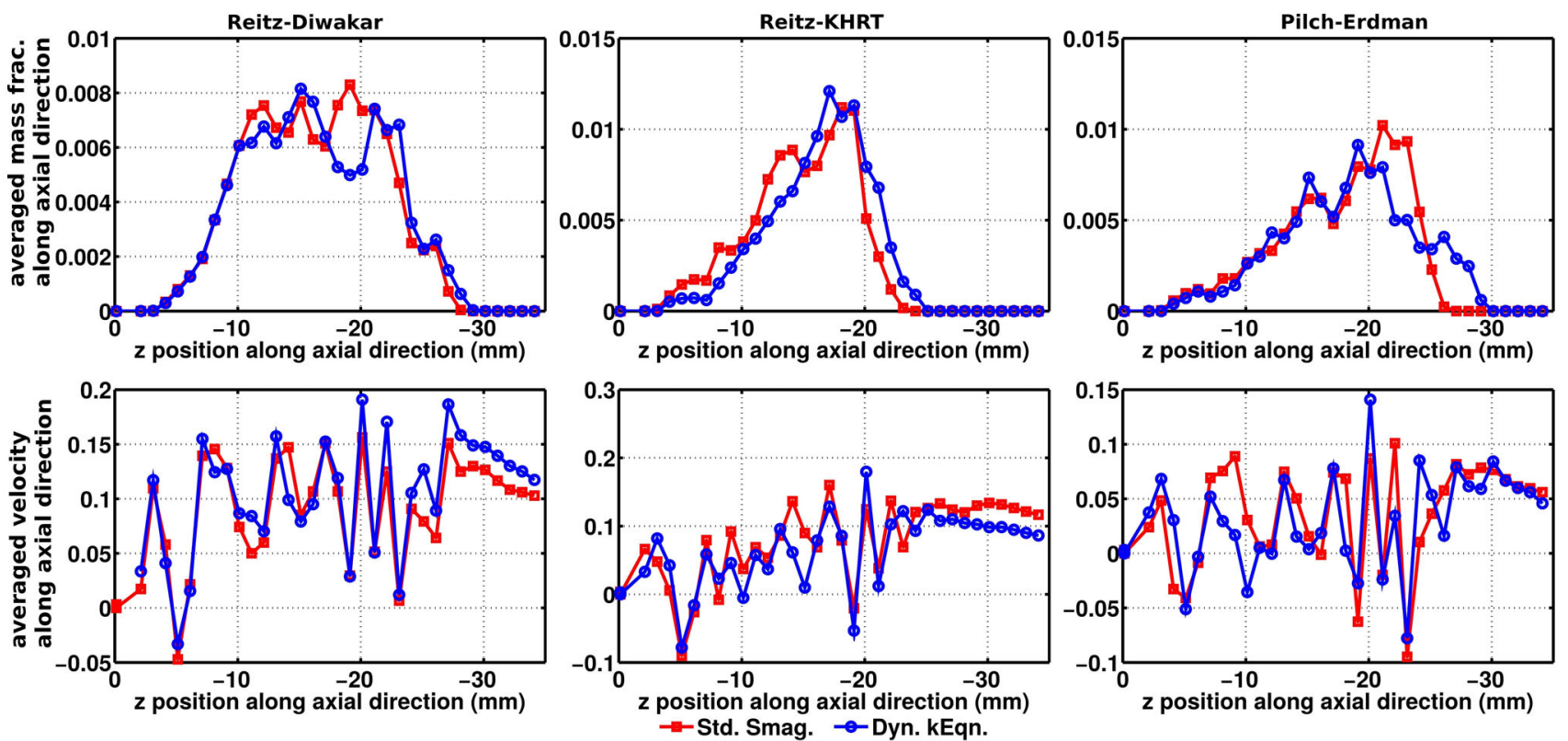

Fig. 26 Profiles of averaged fuel mass fraction and averaged gaseous phase axial velocity at $0.6 \mathrm{~ms}$ after start of injection. Values are averaged over planes at discrete intervals along axial direction (from 0 to $-35 \mathrm{~mm}$ )

simulation, while in the case of liquid penetration length, they were consistent until the end of the injection. Though liquid penetration length reduction was only roughly similar at the end of the injection, the liquid was found to require essentially the same amount of time to evaporate in the simulation and in the experiment, a consistency which reflects the overall quality of the simulation. The set of RANS simulations as a whole was not found to be considerably sensitive to the different resolutions in the range studied, but the LES cases presented more pronounced differences after the end of injection when different grids were used. When the variation types (base, dispersion, and collision) were considered, the inclusion of collision tended to increase the liquid penetration length, sometimes considerably. Substantial changes were also observed when the breakup model was altered; these changes include the tendency toward underprediction during initial instants in the Reitz-KHRT model and poor liquid penetration prediction in the LES/Pilch-Erdman cases.

4. The inclusion of further turbulent dispersion and collision modeling did not alter the results of isooctane mixture fraction more than what would be expected if small statistical fluctuations had been introduced. However, the RANS turbulence framework resulted in a higher probability of finding lean concentrations than the LES framework did. Furthermore, the Reitz-Diwakar was found to produce a slightly higher probability of finding rich fuel mixtures than the other breakup models.

5. No substantial contribution of flow field statistics was found that would justify further modeling of turbulent dispersion and collision. However, the use of different breakup models had a significant effect on the droplet and slip velocity distributions. The results were consistent with the droplet diameter findings (simulations in which a smooth transition from larger to smaller droplets was observed resulted in more spread out and flatter velocity distributions).

6. The very few discrepancies found in the results when comparing the standard Smagorinsky and the dynamic $\mathrm{k}$-Equation subgrid models suggest that the highest resolution used $(0.25 \mathrm{~mm})$ is sufficiently high to capture important features of the complex flow studied, even with a simple algebraic eddy viscosity subgridscale model, as the standard Smagorinsky model.

In future studies, the authors wish to simulate the complete full 8-jet Spray G test case to investigate the effects of jetto-jet interaction, and later, to implement the spray models discussed herein in a complete direct injection engine simulation.

Acknowledgements The authors acknowledge the financial support from the São Paulo Research Foundation, or FAPESP (Case No. 2015/10299-9) and the Energy Department of the São Paulo State University (UNESP), School of Engineering, Guaratinguetá Campus, for providing the computational resources. 


\section{Compliance with ethical standards}

Conflict of interest The authors declare that they have no conflict of interest.

\section{References}

1. ICCT (2014) EU CO2 emission standards for passenger cars and light-commercial vehicles. http://www.theicct.org/sites/default/ files/publications/ICCTupdate_EU-95gram_jan2014.pdf>. Acc essed 26 Oct 2016

2. Goryntsev D, Sadiki A, Klein M, Janicka J (2010) Analysis of cyclic variations of liquid fuel-air mixing processes in a realistic DISI IC-engine using Large Eddy Simulation. Int J Heat Fluid Flow 31:845-849

3. Peterson B, Reuss DL, Sick V (2014) On the ignition and flame development in a spray-guided direct-injection spark-ignition engine. Combust Flame 161:240-255

4. C Baumgarten (2005) Mixture formation in internal combustion engines. Springer, Berlin, Heidelberg

5. Fajgenbaum R, Gonçalves dos Santos R (2016) Influence of fuel temperature on atomization parameters in a pressure-swirl atomizer from a port fuel injector by shadowgraphy technique. J Braz Soc Mech Sci Eng 38(7):1877-1892. https://doi.org/10. 1007/s40430-015-0443-0

6. Yang J, Anderson R (1998) Fuel injection strategies to increase full-load torque output of a direct-injection SI engine. SAE Technical Paper 980495. https://doi.org/10.4271/980495

7. Zheng Z, Tian X, Zhang X (2015) Effects of split injection proportion and the second injection time on the mixture formation in a GDI engine under catalyst heating mode using stratified charge strategy. Appl Therm Eng 84:237-245

8. Manin J, Bardi M, Pickett L, Dahms R, Oefelein J (2014) Microscopic investigation of the atomization and mixing processes of diesel sprays injected into high pressure and temperature environments. Fuel 134:531-543

9. Skeen SA, Manin J, Pickett LM (2015) Simultaneous formaldehyde PLIF and high-speed schlieren imaging for ignition visualization in high-pressure spray flames. Proc Combust Inst 35:3167-3174

10. Westbrook CK, Mizobuchi Y, Poinsot TJ, Smith PJ, Warnatz J (2005) Computational combustion. Proc Combust Inst 30:125-157

11. Vuorinen VA, Hillamo H, Kaario O, Nuutinen M, Larmi M, Fuchs L (2011) Effect of droplet size and atomization on spray formation: a priori study using large-Eddy simulation. Flow Turbul Combust 86:533-561

12. Vuorinen VA, Hillamo H, Kaario O, Larmi M, Fuchs L (2010) Large Eddy simulation of droplets stokes number effects on turbulent spray shape. Atomization Sprays 20:93-114

13. Vuorinen VA, Hillamo H, Kaario O, Nuutinen M, Larmi M, Fuchs L (2010) Large Eddy simulation of droplets stokes number effects on mixture quality in fuel sprays. Atomization Sprays 20:435-451

14. Lucchini T, D'Errico G, Ettorre D (2011) Numerical investigation of the spray-mesh-turbulence interactions for high-pressure, evaporating sprays at engine conditions. Int $\mathbf{J}$ Heat Fluid Flow 32:285-297

15. Jangi M, Solsjo R, Johansson B, Bai X (2015) On large eddy simulation of diesel spray for internal combustion engines. Int $\mathbf{J}$ Heat Fluid Flow 53:68-80

16. Subramaniam S (2013) Lagrangian-Eulerian methods for multiphase flows. Prog Energy Combust Sci 39:215-245
17. Pope SB (2011) Simple models of turbulent flows. Phys Fluids 23(011301):1-20

18. Alkidas AC (1999) Combustion-chamber crevices: the major source of engine-out hydrocarbon emissions under fully warmed conditions. Prog Energy Combust Sci 25:253-273

19. T Nguyen, P Janas, T Lucchini, G D'Errico, S Kaiser, A Kempf (2014) LES of flow processes in an SI engine using two approaches: openfoam and PsiPhi, SAE technical paper 2014-011121. https://doi.org/10.4271/2014-01-1121

20. P Janas, M Ribeiro, A Kempf, M Schild, S Kaiser (2015) Penetration of the flame into the top-land Crevice-Large-Eddy simulation and experimental high-speed visualization, SAE technical paper 2015-01-1907. https://doi.org/10.4271/2015-01-1907

21. A-H Kakaee, M Keshavarz (2017) Simultaneous dynamic optimization of valves timing and waste gate to improve the load step transient response of a turbocharged spark ignition engine. J Braz Soc Mech Sci Eng. https://doi.org/10.1007/s40430-017-0786-9

22. Raggi MVK, Sodré JR (2014) Numerical simulation of carbon monoxide emissions from spark ignition engines. J Braz Soc Mech Sci Eng 36(1):37-43. https://doi.org/10.1007/s40430-0130076-0

23. A Misdariis, O Vermorel, T Poinsot (2015) A methodology based on reduced schemes to compute autoignition and propagation in internal combustion engines. Proc Combust Inst 35:3001-3008

24. SB Pope (2000) Turbulent flows. Cambridge University Press, New York

25. Marshall WR, Ranz WE (1952) Internal combustion engine modeling. Hemisphere Publishing, New York

26. Zuo B, Gomes AM, Rutland CJ (2000) Modelling superheated fuel sprays and vaporization. Int J Engine Res 1:321-336

27. Adachi M, McDonell V, Tanaka D, Senda J, et al. (1997) Characterization of fuel vapor concentration inside a flash boiling spray. SAE Technical Paper 970871. https://doi.org/10.4271/ 970871

28. Reitz RD, Bracco FV (1986) The encyclopedia of fluid mechanics. Gulf Publishing, Houston

29. Wierzba A (1990) Deformation and breakup of liquid drops in a gas stream at nearly critical Weber numbers. Exp Fluids 9:59-64

30. Reitz RD (1987) Modeling atomization processes in high-pressure vaporizing sprays. Atomisation Spray Technol 3:309-337

31. Reitz R, Diwakar R (1987) Structure of high-pressure fuel sprays. SAE Technical Paper 870598. https://doi.org/10.4271/870598

32. Beale JC, Reitz RD (1999) Modeling spray atomization with the Kelvin-Helmholtz/Rayleigh-Taylor hybrid model. Atomization Sprays 9:623-650

33. Pilch M, Erdman CA (1987) Use of breakup time data and velocity history data to predict the maximum size of stable fragments for acceleration-induced breakup of a liquid drop. Int $\mathbf{J}$ Multiphase Flow 13:741-757

34. Arcoumanis C, Gavaises M, French B (1997) Effect of fuel injection processes on the structure of diesel sprays. SAE Technical Paper 970799. https://doi.org/10.4271/970799

35. O'Rourke PJ (1989) Statistical properties and numerical implementation of a model for droplet dispersion in a turbulent gas. J Comput Phys 83:345-360

36. PJ O'Rourke (1981) Collective drop effects on vaporizing liquid sprays, Ph.D. thesis, Princenton University, New Jersey

37. ECN (2016) Spray g operation conditions. http://www.sandia. gov/ecn/G/targetCondition/sprayG.php. Accessed 27 Oct 2016

38. ECN (2014) Ecn 3rd workshop proceedings. http://www.sandia. gov/ecn/workshop/ECN3.php. Accessed 27 Oct 2016

39. Kim W-W, Menon S (1995) A new dynamic one-equation subgrid-scale model for large eddy Simulations. In: 33rd aerospace sciences meeting and exhibit, Reno, NV, USA. https://doi.org/10. 2514/6.1995-356 